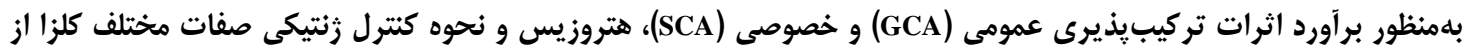

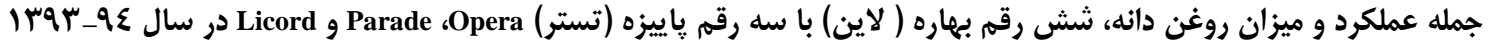
تلاقى داده شدند.

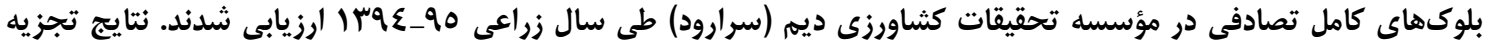

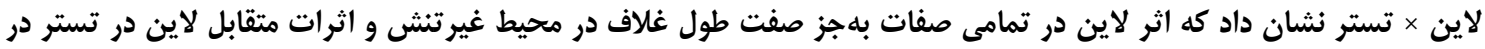

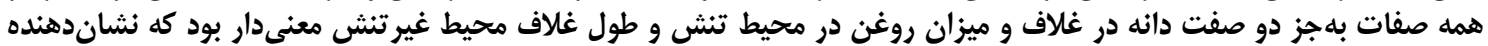

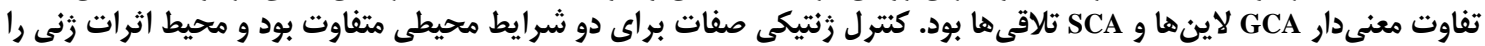

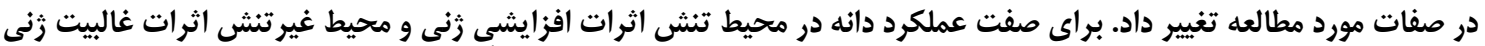

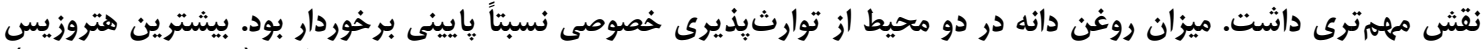

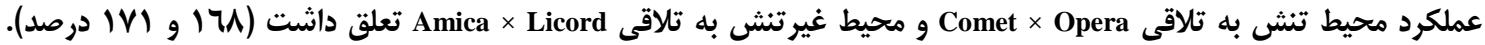

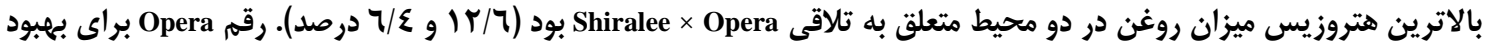

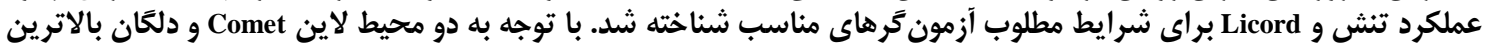

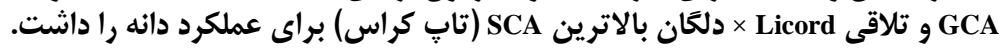

وازههاى كليدى: تركيبذٍيرى، عمل زن، وراثتيذيرى، هتروزيس

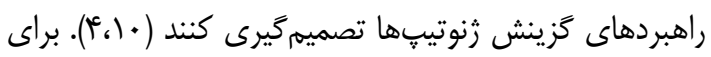

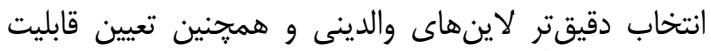

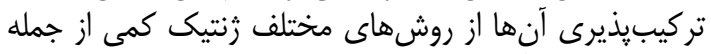

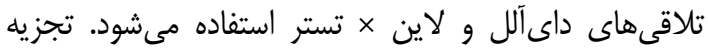

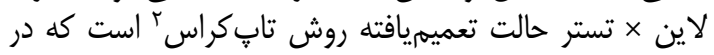

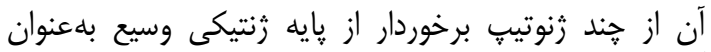

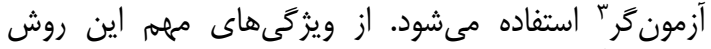

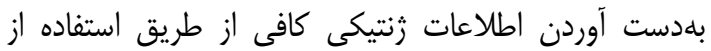

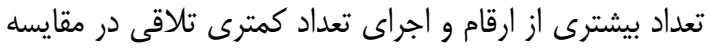

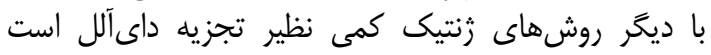

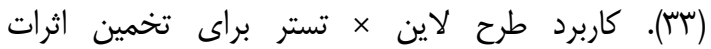

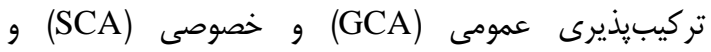

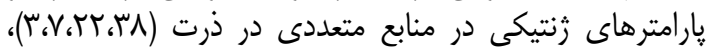

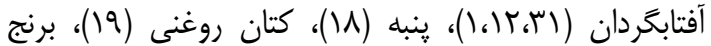

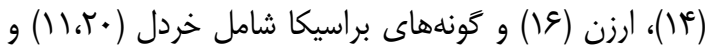

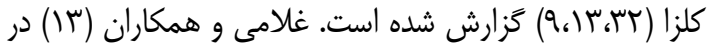

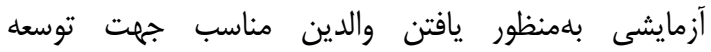

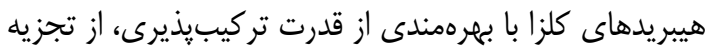

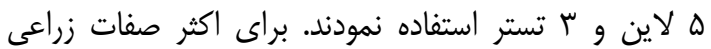

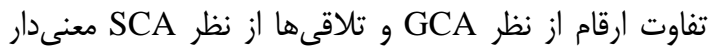

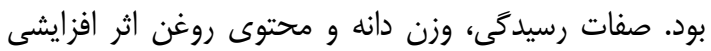

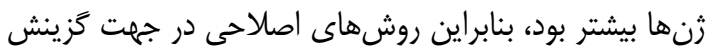

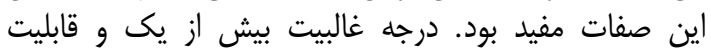

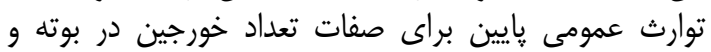

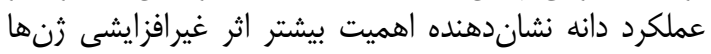

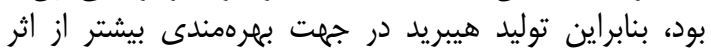

مقدمه كلزا (Brassica napus L.) يكى از مارهمترين كياهان

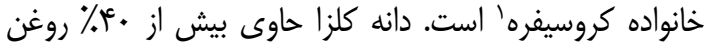

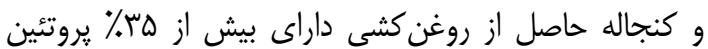

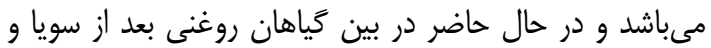

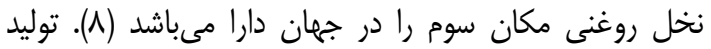

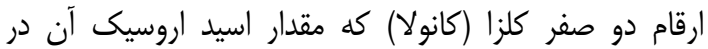

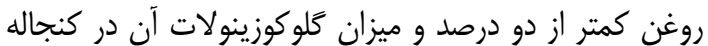

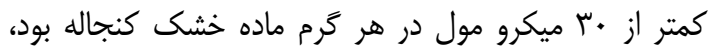

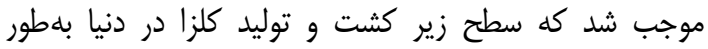

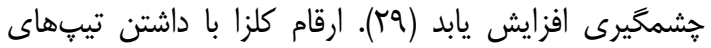

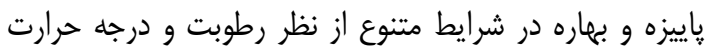

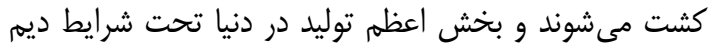

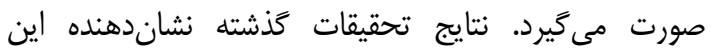

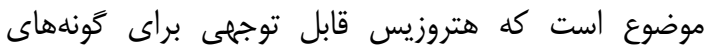

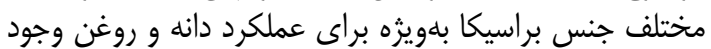

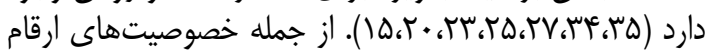

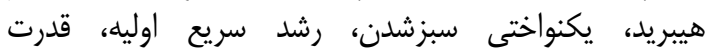

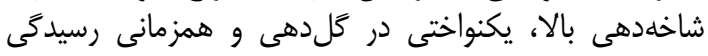

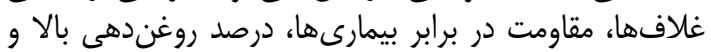

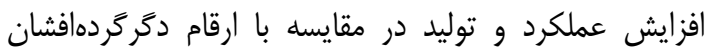

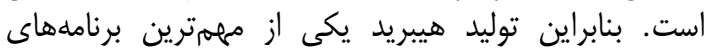

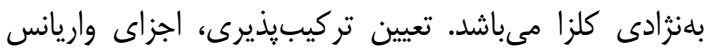

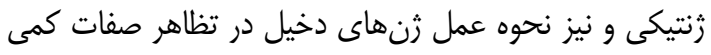

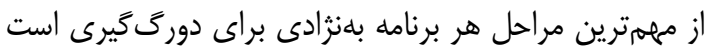

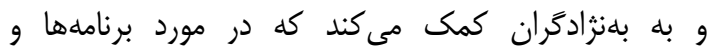


تا رسيدگى، ارتفاع گياه، تعداد شاخه فرعى در هر بوته، تعداد

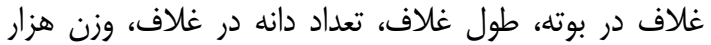

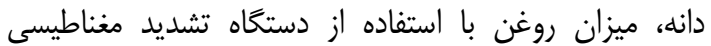

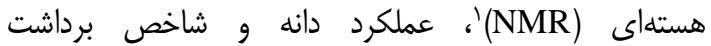

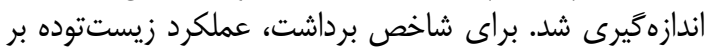

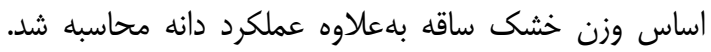

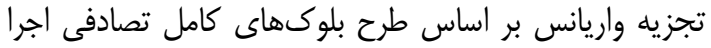

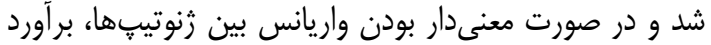

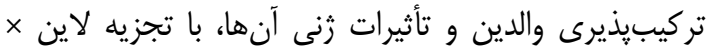

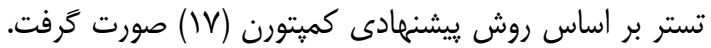

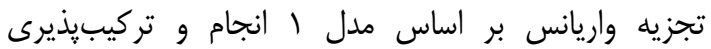
عمومى و خصوصى لاينها و رونس تسترها تعيين شد: $Y_{\mathrm{ij}}=\mu+\mathrm{g}_{\mathrm{ii}}+g_{j j}+s_{i j}+r_{k}+e_{i j k}$ رابطه (1) در اين رابطه، Yij: ميانگين فنوتييى اندازهيرى شده

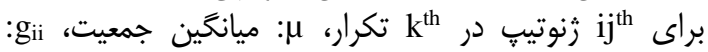

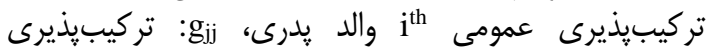

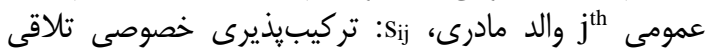

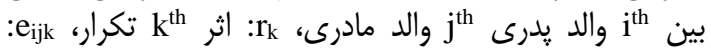

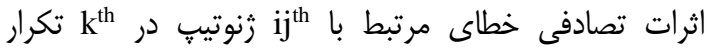

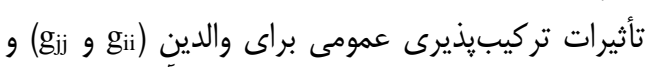
مىباشند.

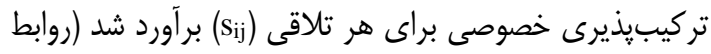

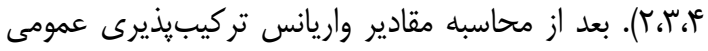

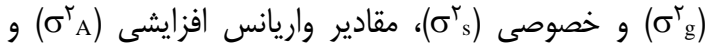

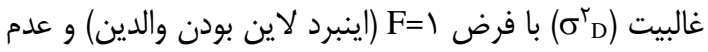

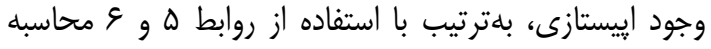

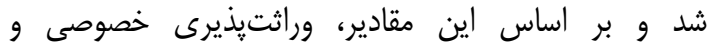

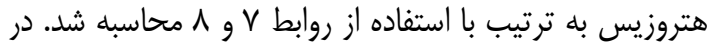

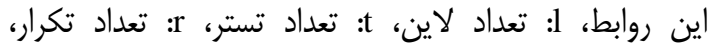
مربات

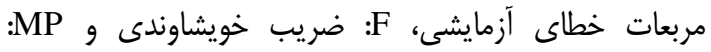
ميانخين والدين (لاينها و تسترها) هر تلاقى است (باتئ (r).

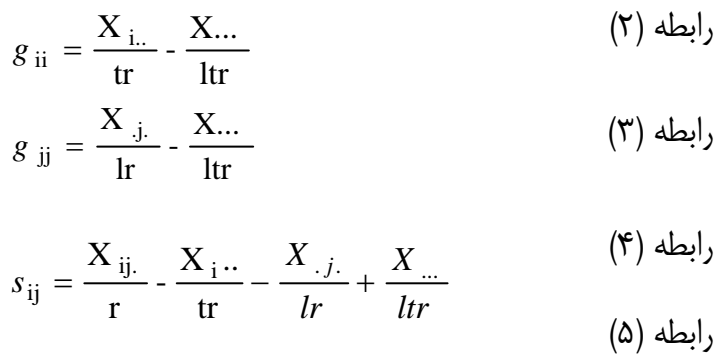

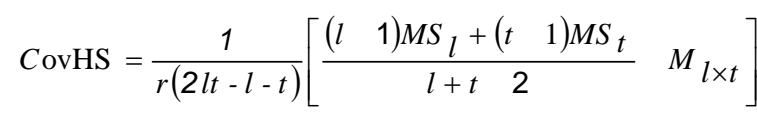

$\sigma_{\mathrm{sca}}^{2}=\frac{M S_{l \times t}-M S_{e}}{r}=\left(\frac{1+\mathrm{F}}{2}\right)^{2} \sigma_{\mathrm{D}}^{2}$
غيرافزايشى ثنهاى كنترل كننده اين صفات قابل توجيه شد.

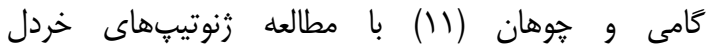
(B. juncea)

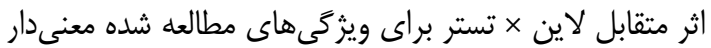

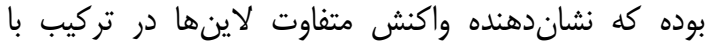

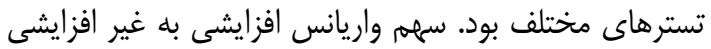

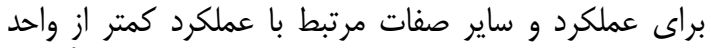

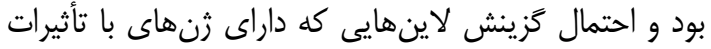

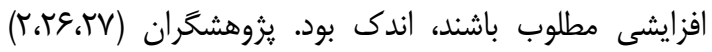

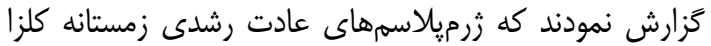

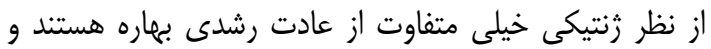

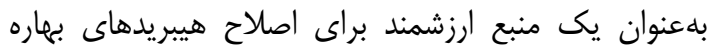

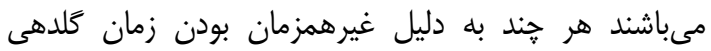

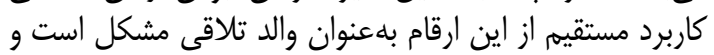

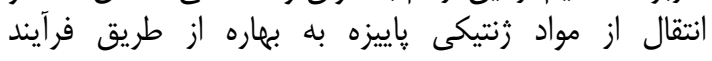

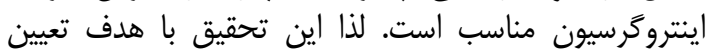

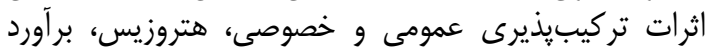

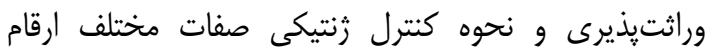

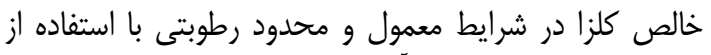

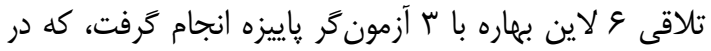

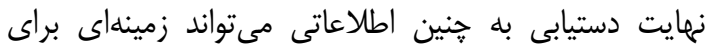
انتخاب روش اصلاحى مناسب در هر دو شرايط باشئ داشئ

\section{مواد و روشها}

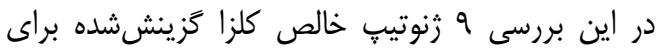

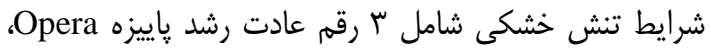

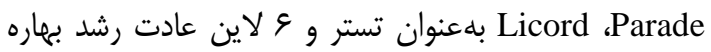

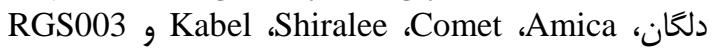

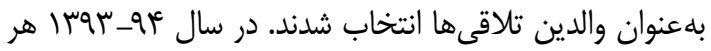

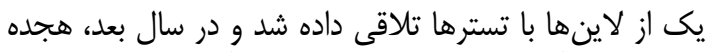

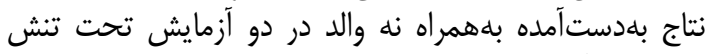

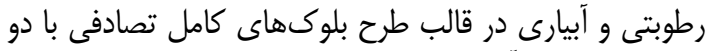

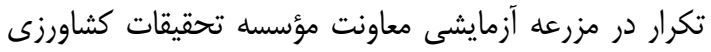

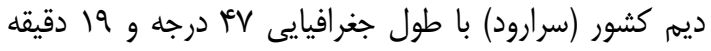

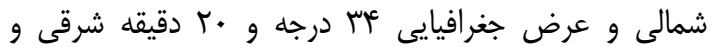

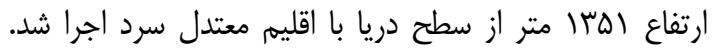

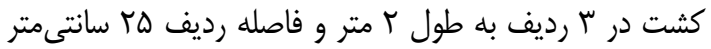

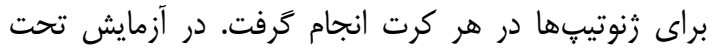

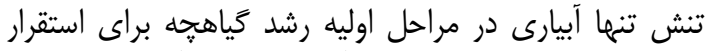

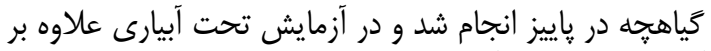

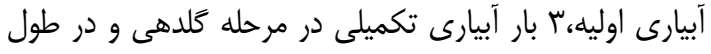

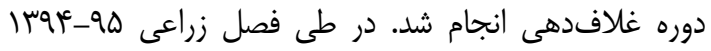

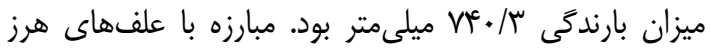

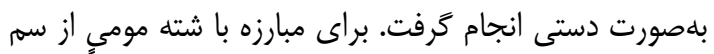

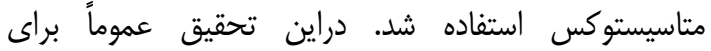

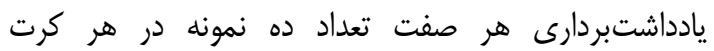

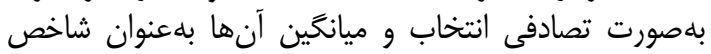

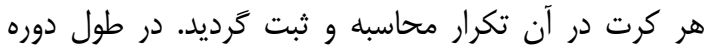

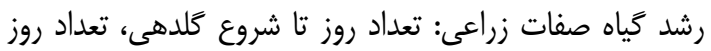




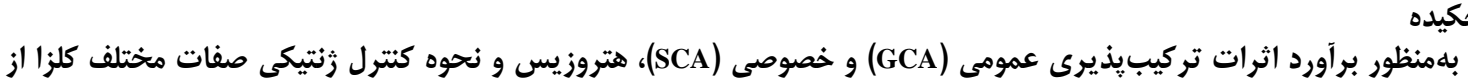

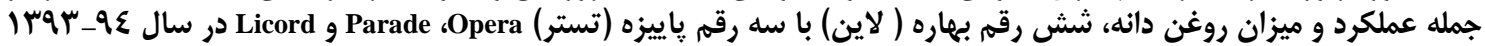

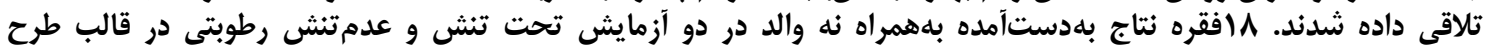

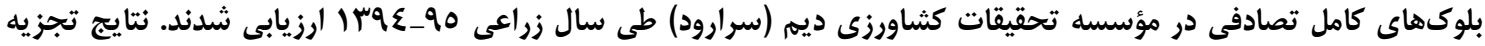

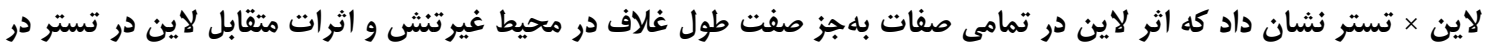

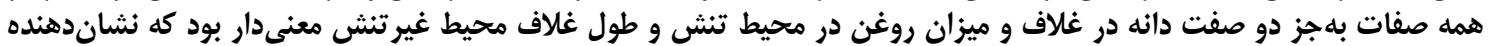

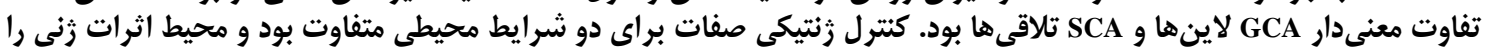

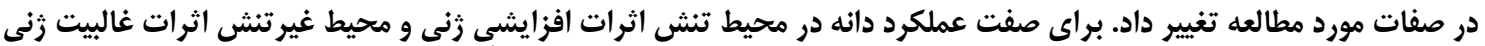

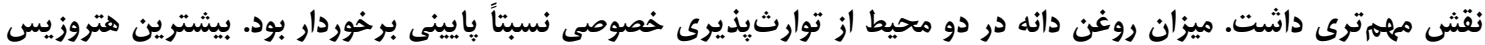

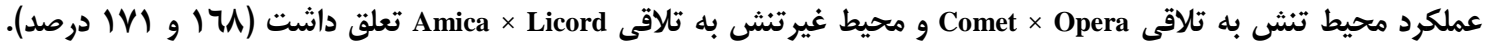

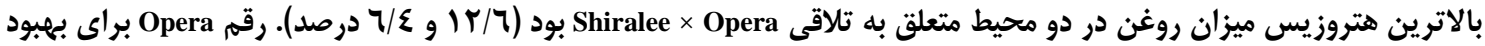

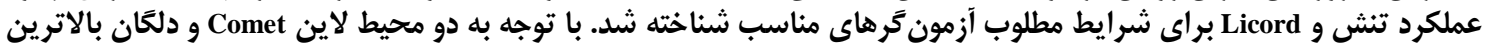

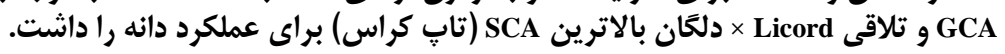

وازههاى كليدى: تركيبذيذي، عمل زن، وراثتيذيرى، هتروزيس

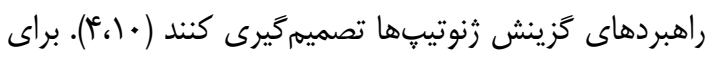

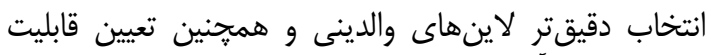

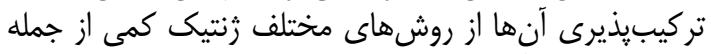

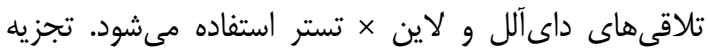

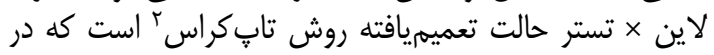

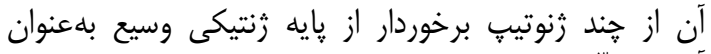

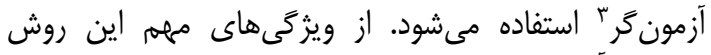

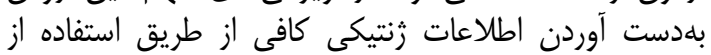

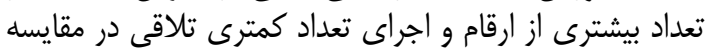

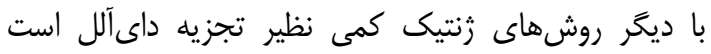

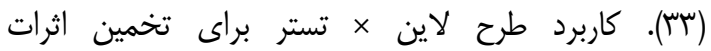

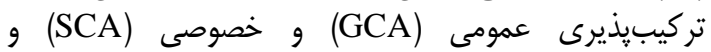

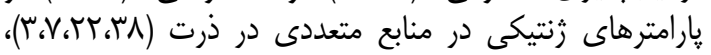

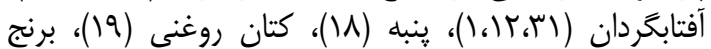

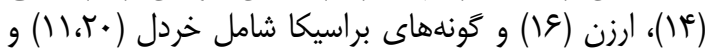

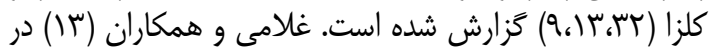

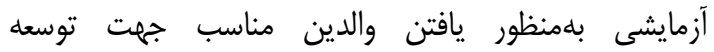

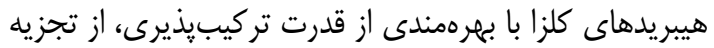

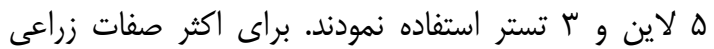

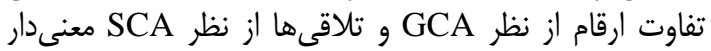

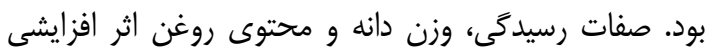

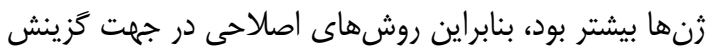

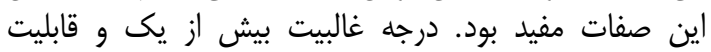

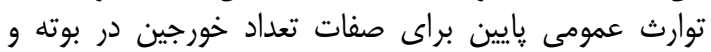

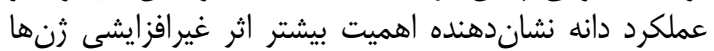

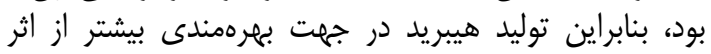

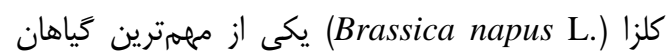

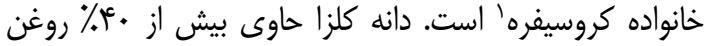

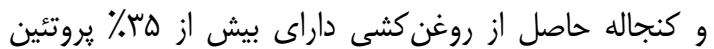

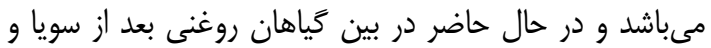

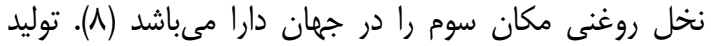

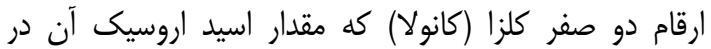

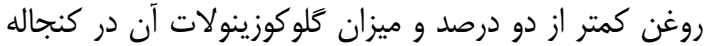

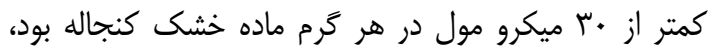

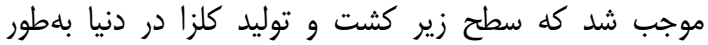

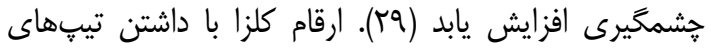

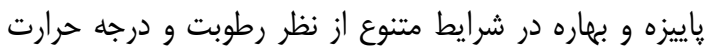

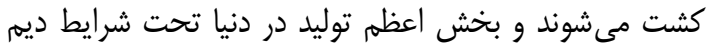

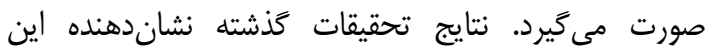

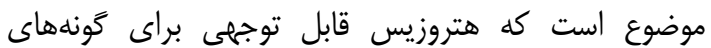

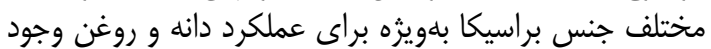

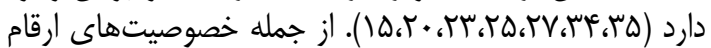

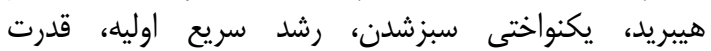

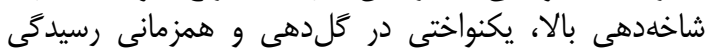

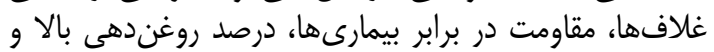

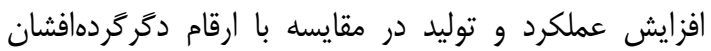

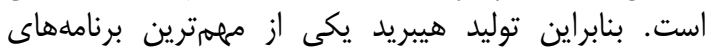

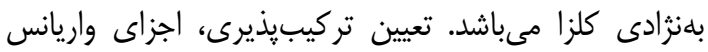

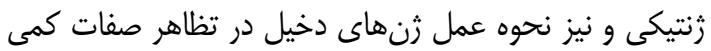

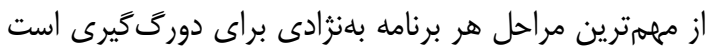

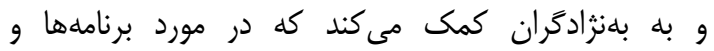


غلاف در هر دو شرايط و ميزان روغن در شرايط تنش براى

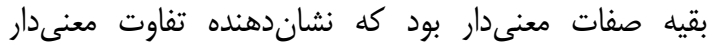

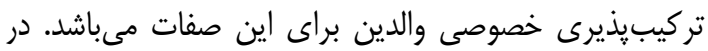

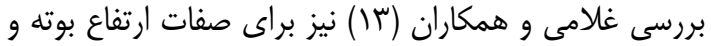

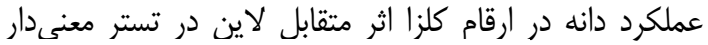

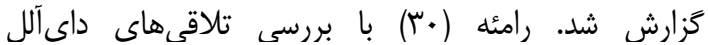

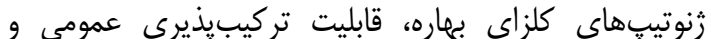

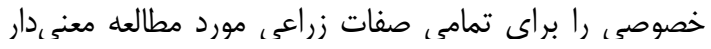

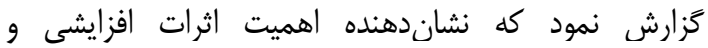
غيرافزايشى زن هادي در كنترل اين صفات مى دياشد.

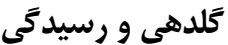
بر اساس آزمون تركيبيذيرى براى صفاى صفات مختلف،

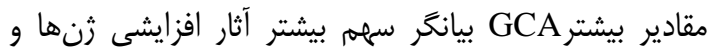

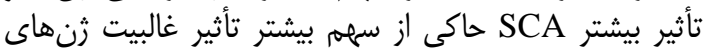

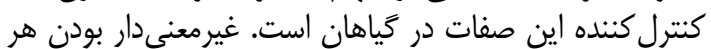

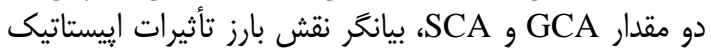

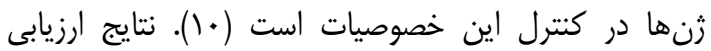

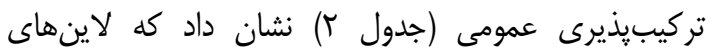

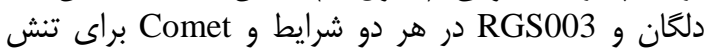

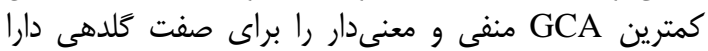

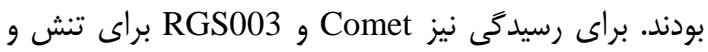

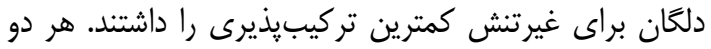

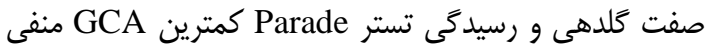

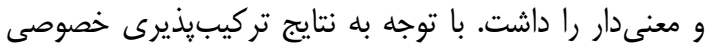

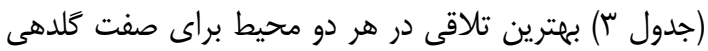

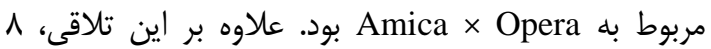

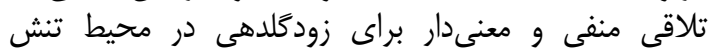

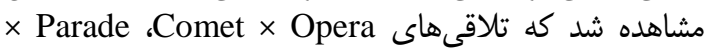

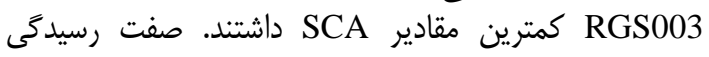

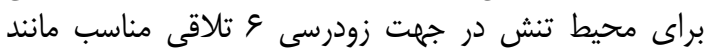
Comet × Opera و Amica × Parade

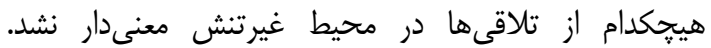

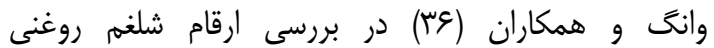
تركيد (Brassica rapa)

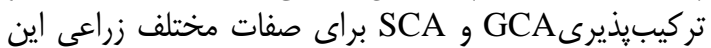

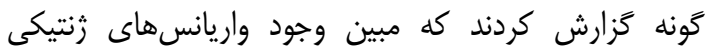

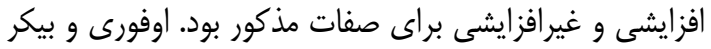

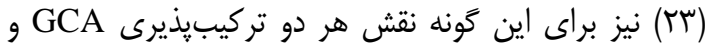

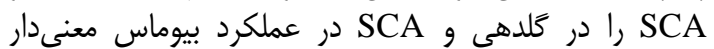

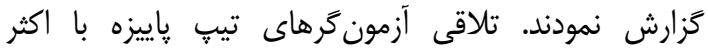

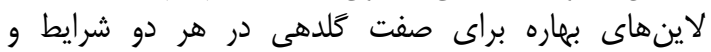

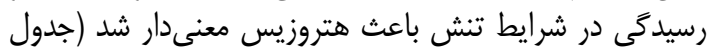

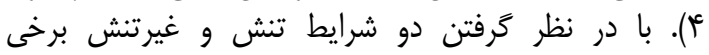

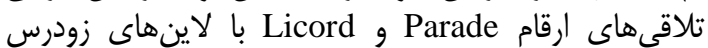

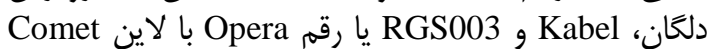

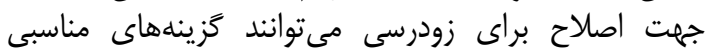

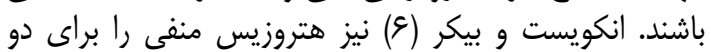

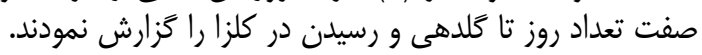

$$
\begin{aligned}
& \sigma_{\mathrm{gca}}^{2}=\operatorname{CovHS}=\left(\frac{1+F}{4}\right) \sigma_{\mathrm{A}}^{2} \quad \text { (V) } \\
& H_{\mathrm{n}}=\frac{\sigma_{\mathrm{A}}^{2}}{\sigma_{\mathrm{A}}^{2}+\sigma_{\mathrm{D}}^{2}+\sigma_{\mathrm{e}}^{2} / r} \quad \text { (ابطه } \\
& H_{\mathrm{et}}=\frac{\left(F_{1}-M P\right)}{M P} \times 100 \quad \text { (9) }
\end{aligned}
$$

نتايج و بحث

نتايج تجزيه واريانس مجزا دادههاى دو محيط تحت تنش

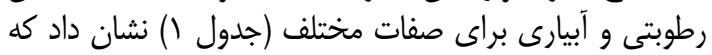
اثر تيمار بهجز صفت دانه در غلاف برات براى شرايط آبيارى برائ براى

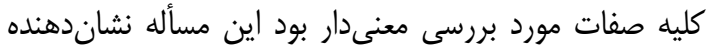

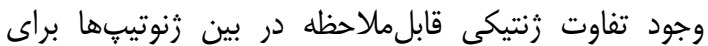

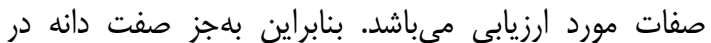

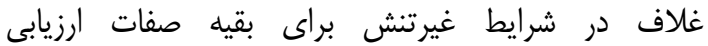

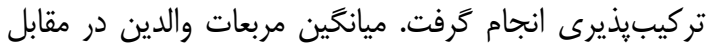

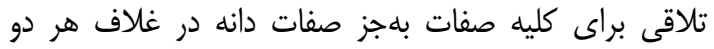

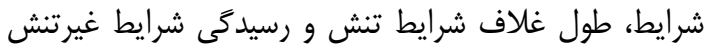

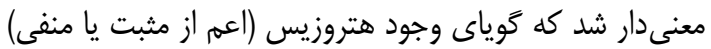

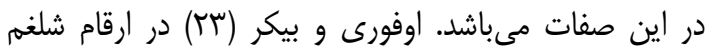

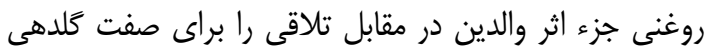

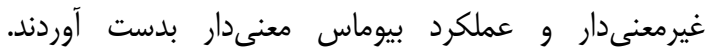

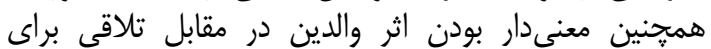

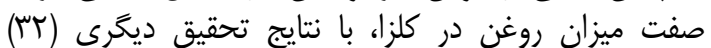

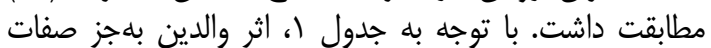

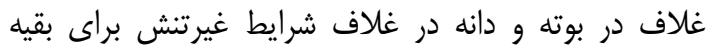

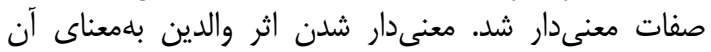

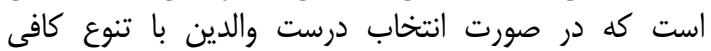
مىتوان مقدار صفت در هيبريد را به سمت داب دلخ الخواه سوق داد داد.

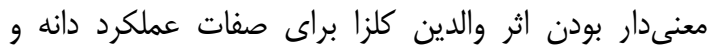

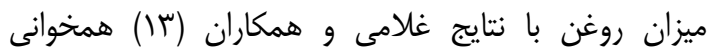

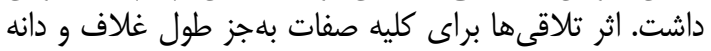

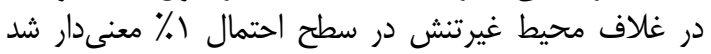

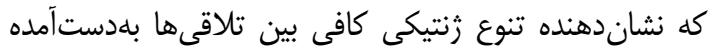

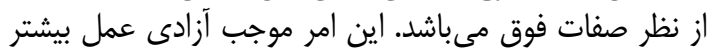

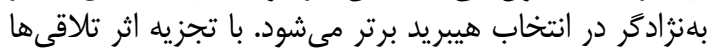

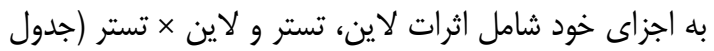

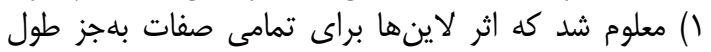

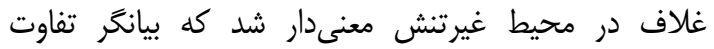

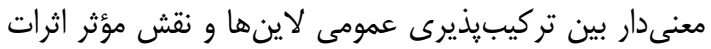

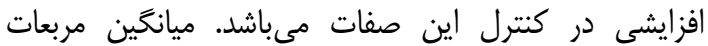

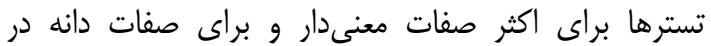

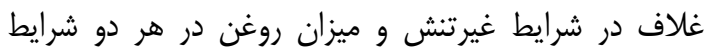

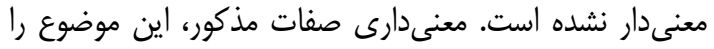

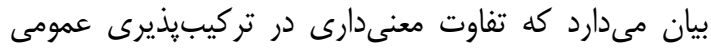

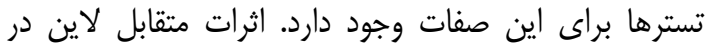

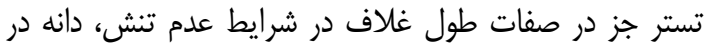




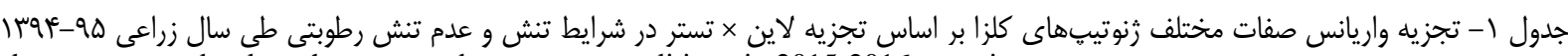
Table 1. Line $\times$ tester Analysis for different traits in rapeseed genotypes under drought stress and non stress conditions in 2015 -2016 cropping season

\begin{tabular}{|c|c|c|c|c|c|c|c|c|c|c|c|}
\hline \multicolumn{2}{|c|}{ طول غلاف } & \multicolumn{2}{|c|}{ غاف در بوته } & \multicolumn{2}{|c|}{ ارتفاع بوته } & \multicolumn{2}{|c|}{ رسيدگى } & \multicolumn{2}{|c|}{ كلدهى | ملدي } & \multirow{2}{*}{ درجه آزادى } & \multirow{2}{*}{ منبع تغييرات } \\
\hline غيرتنش & تنش & غيرتنش & تنش & غيرتنش & تنش & غيرتنش & تنش & 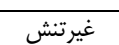 & تنش & & \\
\hline.$/ 4 \varepsilon^{*}$ &.$/ 9 \vee q^{* *}$ & $11 \% \cdot / 11^{* *}$ & $r \cdot \Lambda \Delta / \mu \xi^{* *}$ & GTN/Tr"** & $r r \cdot \Delta / V)^{* *}$ & $f \cdot / f v^{* *}$ & $g g / \pi \omega^{* *}$ & $r \cdot 9 / 1 .^{* * *}$ & $r \cdot \Delta / \Lambda)^{* *}$ & Tq & تيمار \\
\hline$\cdot|r| *$ & $\cdot / 9 \Lambda^{* *}$ & $\Delta Q \mathcal{N} / \Delta T^{n s}$ & $|r| V / \mid F^{* *}$ & $\Lambda \varepsilon \cdot /{ }^{* * *}$ & $r V \cdot 9 / 9)^{* *}$ & 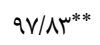 & $|Q| / \wedge 9^{* *}$ & $\Delta \Delta \Psi / / \Gamma^{* * *}$ & $\kappa \kappa r / v Q^{* *}$ & $\wedge$ & والدين \\
\hline 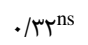 & $\cdot / \Delta V \Delta^{* *}$ & $\mid r A T / \cdot e^{* * *}$ & $r \mid V \varepsilon / \cdot r^{* *}$ & $r \Delta \cdot / \cdot q^{* *}$ & $1911 / .^{* *}$ & $\mid \omega / \mathcal{F} \gamma^{* *}$ & rq/rq** & $r N \cdot r^{* * *}$ & $\Lambda \mathcal{N} / \mathcal{F q}^{* *}$ & iv & تلاقى ها \\
\hline . $/ r^{\mathrm{ns}}$ & $1 / r \omega^{* *}$ & $q \vee \cdot / v \cdot * *$ & 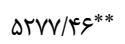 & 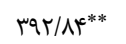 & $\Delta Q G \times / q \Gamma^{* * *}$ & $r q / q^{* *}$ & $\Gamma \Lambda / T \gamma^{* *}$ & $\Delta / / / \Gamma^{* * *}$ & $\Delta V(\Delta)^{* *}$ & $\Delta$ & لاين \\
\hline.$/ 9 \gamma^{* *}$ &.$/ q^{* *}$ & $F \Psi V / T V^{* *}$ & | (צ" & $9 \vee r / \% \omega^{* *}$ & rqR/99** & r $N / 9 q^{* * *}$ & $11 \% / r Q^{* *}$ & $|Q T /|^{* * *}$ & $\Gamma \Delta \omega / \cdot \Lambda^{* *}$ & r & تستر \\
\hline.$/ \mu^{\mathrm{ens}}$ &.$/ 19^{* *}$ & 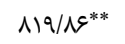 & 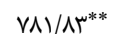 & $r \cdot r / r q^{* *}$ & rTQ/Q.*** & $r / V r^{*}$ & $N / r^{* *}$ & $\Lambda / \Delta \Lambda^{* *}$ & $\mathbb{F} / \Gamma \Delta^{* *}$ & 1. & ل لاين × تستر \\
\hline.$/ 94^{*}$ & $\cdot / \cdot 0^{\mathrm{ns}}$ & $r \Lambda . . / \cdot r^{* * *}$ & $9819 / 9 . * *$ & $r \Delta \cdot . / \cdot \Delta^{* *}$ & $199 \% / \backslash \Lambda^{* *}$ & $r / q q^{n s}$ & $\checkmark / \leftarrow^{*} \Delta^{* *}$ & $r q \Delta / / \Gamma^{* * *}$ & $r \mu / V r^{* * *}$ & 1 & والدين در مقابل تلاقى \\
\hline.$/ 1 F$ &.$/ \cdot \Delta$ & ITr/Tg & $\| f / g F$ & $\mathrm{rr} / \mathrm{qr}$ & $\lceil\mathcal{N} / \Lambda$ & $1 / 9 \Delta$ &.$/ \cdot \Delta$ & 1/9ه & $\cdot / r \Lambda$ & te & خطا \\
\hline V/rg & $F / \Delta F$ & $N / \Lambda)$ & $1.1 \Delta \Delta$ & $r / r$. & $F / W$ &.$/ 01$ & .11 &.$/ \mathrm{V}$ &.$/ \mu F$ & & ضريب تغييرات (\%) \\
\hline
\end{tabular}

Table 1. Continued

ادامه جدول

\begin{tabular}{|c|c|c|c|c|c|c|c|c|c|c|c|}
\hline \multicolumn{10}{|c|}{ ميانكين مربعات صفات مورد بررسى } & \multirow{3}{*}{ درجه آزادى } & \multirow{3}{*}{ منبع تغييرات } \\
\hline \multicolumn{2}{|c|}{ شاخص برداشت } & \multicolumn{2}{|c|}{ عملكرد دانه } & \multicolumn{2}{|c|}{ ميزان روغن دانه } & \multicolumn{2}{|c|}{ وزن هزار دانه } & \multicolumn{2}{|c|}{ دانه در غلاف } & & \\
\hline غيرتنش غ غ & تنش & غيرتنش & تنش & 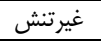 & تنش & 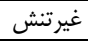 & تنش & 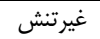 & تنش & & \\
\hline$\Delta \varepsilon / \Lambda r^{* *}$ & $\Lambda \varepsilon / \cdot \Gamma^{* * *}$ & $101 V 9 \& V / 1 . * *$ & $\mid r \mu q \Delta \wedge \Delta / \Lambda r^{* *}$ & $r / \mathrm{r})^{* *}$ & $\mid g / F^{*} V^{* *}$ & $\cdot / \Delta f^{* *}$ & $1 / 4^{* * *}$ & $11 / 9 Y^{\mathrm{ns}}$ & $r \cdot / \wedge q^{* *}$ & rq & تيمار \\
\hline 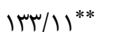 & $|F| / r q^{* *}$ & l & $\Lambda F V \Delta \cdot \Delta / V^{* * *}$ & $0 / .9^{*}$ & $T V / V q^{* *}$ &.$/ \Delta F^{* * *}$ & $1 / v Q^{* *}$ & $\Delta / / r^{\mathrm{ns}}$ & $r \mathrm{r} / \Lambda D^{* *}$ & $\Lambda$ & والدين \\
\hline$T r / T V^{* *}$ & $r Y / \mathcal{Y})^{* *}$ & $9.99 \Lambda . / . .^{* *}$ & $\| \S \xi \cdot \Delta \cdot / \Lambda \Delta^{* *}$ & $\Gamma / \Lambda Q^{* *}$ & $1 . / 18^{* *}$ &.$/ \mu \varphi^{* * *}$ & $1 / .9^{* *}$ & $\mid c / a T^{n s}$ & $\mid \varepsilon / .1^{* *}$ & iv & ت ت تلاقىها \\
\hline ห৭/१०** & $\Lambda r / q^{* * *}$ & $1 Q 9 \cdot r \cdot 1 / / \Lambda^{* *}$ & TYMT.VN/VV** & $r / 9 \Delta^{* *}$ & $r r / q r^{* *}$ &.$/ 99^{* *}$ & $r / 9 r^{* *}$ & $r T / 9 \Delta^{* *}$ & $r T / M e^{* * *}$ & $\Delta$ & لاين \\
\hline$\kappa \kappa / g r^{* *}$ & $1 . / 4 V^{*}$ & 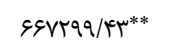 & $\vee Q \& \wedge \Delta \Delta / \Delta r^{* * *}$ & $1 / v c^{n s}$ & $\kappa / \widetilde{L} \Delta^{\mathrm{ns}}$ & $\cdot / \cdot \mathrm{v}^{* *}$ & $\cdot / \Lambda \Delta^{* *}$ & $r \cdot / M^{n s}$ & $\mid F / M^{*}$ & r & تستر \\
\hline $\mid r / q v^{* *}$ & $|T /| \Delta^{* *}$ & gIV $\Delta \Delta \Delta / \Delta r^{* * *}$ & $r \cdot I V V \Delta / ৭ \varphi^{* *}$ & $1 / r^{*}$ & $F / c^{c} e^{n s}$ & $\cdot / r^{* *}$ &.$/ 10^{*}$ & $q / v r^{\mathrm{ns}}$ & $\mathrm{V} / \mathrm{AV} \mathrm{n}^{\mathrm{ns}}$ & 1. & لاين × تستر \\
\hline$\Gamma / \Lambda \omega^{* *}$ & $\Delta D \mathrm{~J} / \tau V^{* *}$ & $|r \cdot q \Delta \Delta \vee N / \Delta|^{* *}$ & DSWRTrY $/ \cdot V^{* *}$ & $19 / \kappa \mu^{* * *}$ & $\mathrm{r} / \Lambda \Lambda^{* *}$ & $r / 9 r^{* *}$ & $r / 9)^{* * *}$ & $V / \Delta T^{n s}$ & $\cdot / / \mathrm{v}^{\mathrm{ns}}$ & 1 & والدين در مقابل تلاقى \\
\hline$r / .9$ & $r / r r$ & rimar/qu & feVeq/r. &.$/ D F$ & $r / r V$ &.$/ .1$ &.$/ \cdot v$ & s/Vr & $r / N^{e}$ & q & خطا \\
\hline V/M & $r / q)$ & $V / F V$ & $1 . / 14$ & V/aV & ו ו & $r / 9 V$ & ת/q & $1 \% /$. & $9 / M$ & & 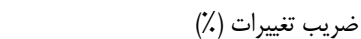 \\
\hline
\end{tabular}


جدول r- قابليت تر كيبزيذيرى عمومى (GCA) لاينها و تسترها براى صفات زراعى ارقام كلزا در شرايط تنش و عدم تنش رطوبتى Table 2. The general combining ability (GCA) of lines and testers for agronomical traits of rapeseed varieties under drought stress and non stress conditions

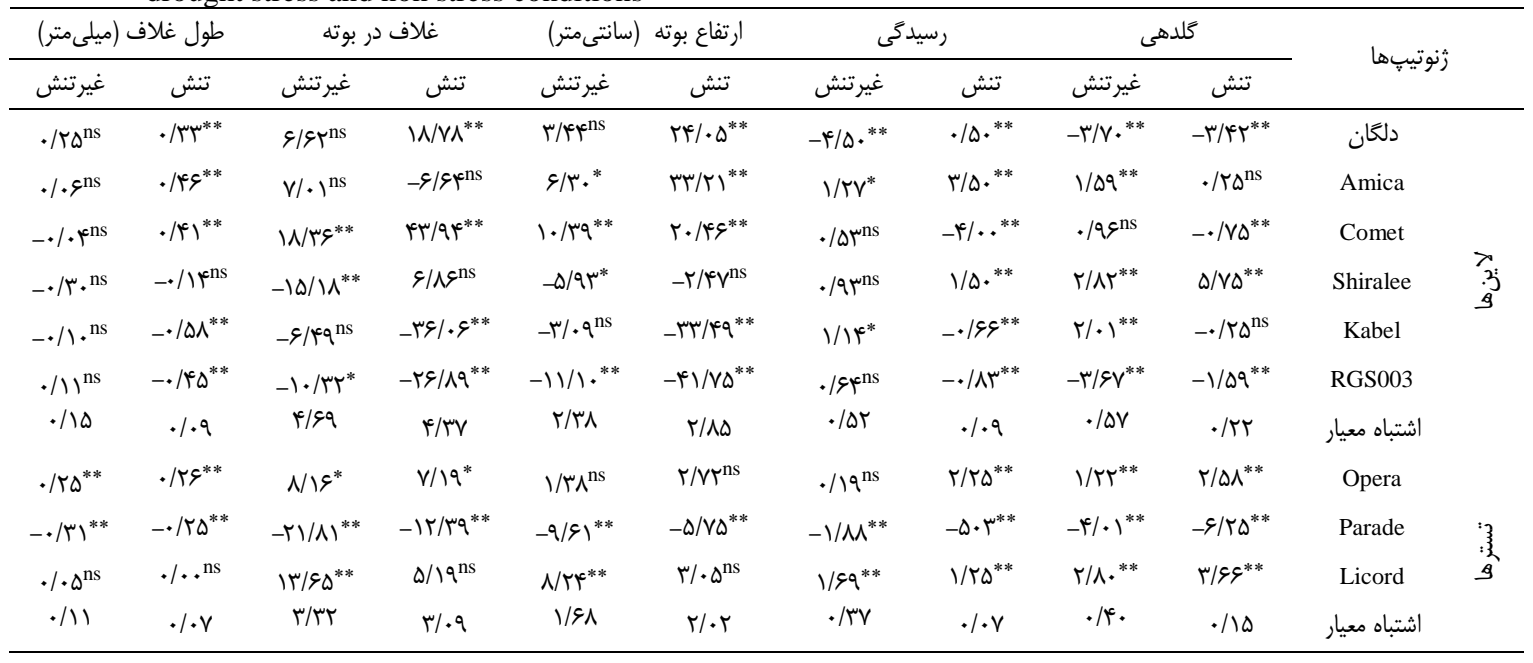

Table 2. Continued

ادامه جدول r

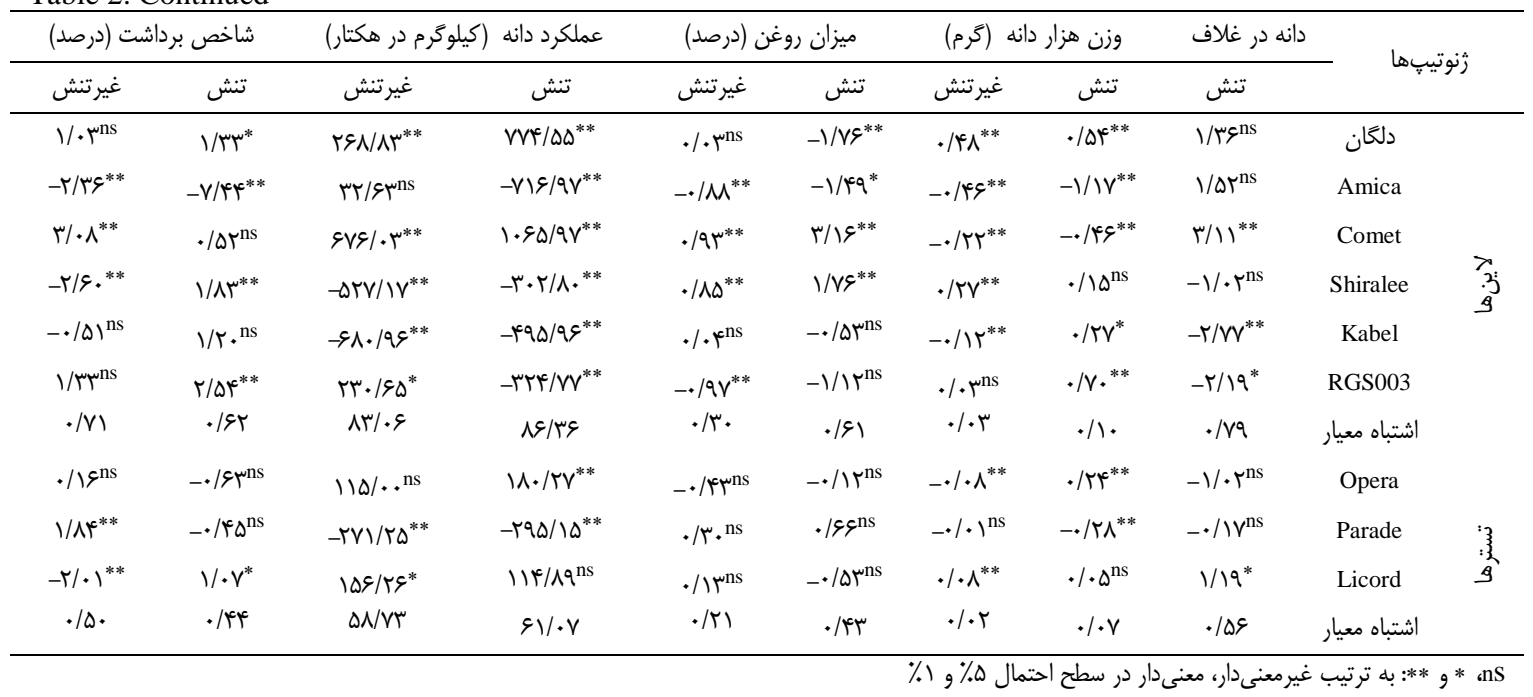


جدول س- قابليت تركيبزيذيرى خصوصى (SCA) تلاقىها (و لاين × س تستر) براى صفات زراعى زنوتيٍهاى كلزا در شرايط تنش و عدم تنش رطوبتى

Table 3. The specific combining ability (SCA) of the crosses ( 6 lines $\times 3$ testers) for agronomic traits of rapeseed

\begin{tabular}{|c|c|c|c|c|c|c|c|c|c|c|c|}
\hline \multicolumn{2}{|c|}{ طول غلاف } & \multicolumn{2}{|c|}{ غلاف در بوته } & \multicolumn{2}{|c|}{ ارتفاع بوته } & \multicolumn{2}{|c|}{ رسيدگى } & \multicolumn{2}{|c|}{ كلدهى } & \multirow[b]{2}{*}{ لاينها } & \multirow[b]{2}{*}{ تستر } \\
\hline غيرتنش & تنش & غيرتنش & تنش & غيرتنش & تنش & غيرتنش & تنش & غيرتنش & تنش & & \\
\hline$-\cdot \cdot / \cdot 1^{\mathrm{ns}}$ & $\cdot / r \omega^{\mathrm{ns}}$ & $1 \cdot / \mu^{\mathrm{ns}}$ & $|f / g|^{\mathrm{ns}}$ & $|r / \Delta|^{* *}$ & $1 / \Gamma \Delta^{\mathrm{ns}}$ & $-1 / \wedge \mu^{n s}$ & $\cdot / \uparrow^{* * *}$ & $-\cdot / r \cdot{ }^{\mathrm{ns}}$ & g/fr** & دلكان & \\
\hline . & $\cdot \mid \mu^{n s}$ & $1 \cdot / 1 \cdot \mathrm{ns}$ & $1.1 .9^{\mathrm{ns}}$ & $\varphi / q \Lambda^{\mathrm{ns}}$ & $-r / \cdot q^{\mathrm{ns}}$ & $-\cdot / 0 \cdot{ }^{\mathrm{ns}}$ & $|/ 4|^{* *}$ & $-r / V \cdot *$ & $-\Delta / r \Delta^{* *}$ & Amica & \\
\hline$-\cdot / 1 \varepsilon^{\mathrm{ns}}$ & $\cdot / / \mathrm{ens}^{\mathrm{ns}}$ & $-V / V^{w^{n s}}$ & $-\varepsilon / V \Lambda^{\mathrm{ns}}$ & $-r / \Delta V^{n s}$ & $-\mu / v \Delta^{\mathrm{ns}}$ &.$- / 8 v^{n s}$ & $-\Gamma / \Delta Q^{* *}$ & $\cdot / \mathcal{A} V^{\mathrm{ns}}$ & $-g / T \Delta^{* *}$ & Comet & $\frac{\pi}{0}$ \\
\hline$-\cdot 1 \cdot 9^{\mathrm{ns}}$ & $-\cdot / \cdot 9^{\mathrm{ns}}$ & $-1 / / Q^{\mathrm{ns}}$ & $-\mid r / 4 \varphi^{c n s}$ & $-\cdot / \& \omega^{\mathrm{ns}}$ & $11 / r \Delta^{*}$ & Wrns & $-1 / \Delta q^{* *}$ & $-1 / r \cdot{ }^{n s}$ & $-1 / V \Delta^{* *}$ & Shiralee & ठ้ \\
\hline$\cdot / \cdot 0^{\mathrm{ns}}$ & $-\cdot / r \cdot{ }^{\mathrm{ns}}$ & $-\mid \varepsilon / q^{\mathrm{ns}}$ & $\Delta / r r^{\mathrm{ns}}$ & $-ه / Y F^{\mathrm{ns}}$ & $-\mu / \mu F^{n s}$ & $1 / \Delta \cdot{ }^{\mathrm{ns}}$ & $1 / \Delta \Lambda^{* *}$ & $r / 9 V^{* *}$ & $r / v \Delta^{* *}$ & Kabel & \\
\hline$-\cdot / \cdot 1^{\mathrm{ns}}$ & $-\cdot / \kappa \Delta^{* *}$ & $r / / r^{n s}$ & $-q / 9 q^{n s}$ & $-V / N e^{\mathrm{ns}}$ & $-r / r)^{n s}$ & $\cdot / \mathrm{v}^{\mathrm{ns}}$ & $\cdot / v \Delta^{* *}$ & $\cdot \mid g^{\mathrm{ens}}$ & $r / . q^{* *}$ & RGS003 & \\
\hline$-\cdot / \Delta Y^{\mathrm{ns}}$ & $-\cdot / \mu^{n s}$ & - & $-\Gamma r / V \Lambda^{* *}$ & $-\mid V / 9 q^{* *}$ & $-r \mid \in \varphi^{* *}$ & $\cdot / \mathrm{V}^{\mathrm{ns}}$ & $1 / 18^{* *}$ & $-1 / \xi^{n s}$ & $-r / V \Delta^{* *}$ & دلغان & \\
\hline$-\cdot / 19^{\mathrm{ns}}$ & ./. rns & $-1 / q \mathrm{Y}^{\mathrm{ns}}$ & $-r / \xi^{n s}$ & $-\varepsilon / \Lambda r^{\mathrm{ns}}$ & $-\varepsilon / \cdot \Delta^{\mathrm{ns}}$ & $\cdot / \Delta \Lambda^{\mathrm{ns}}$ & $-r / \Lambda r^{* * *}$ & $1 / 9 e^{\mathrm{ns}}$ & $r / \bullet^{* *}$ & Amica & \\
\hline$\cdot / / Q^{\mathrm{ns}}$ & $-* / r q^{n s}$ & $\Lambda / \Lambda \cdot n s$ & $-r / \& q^{n s}$ & $\Delta / \mathcal{F r}^{\mathrm{ns}}$ & $V / r q^{n s}$ & . $/ \mathrm{Frns}^{\mathrm{ns}}$ & $1 / 99^{* *}$ & $\cdot / \mu \cdot n s$ & $ץ / \bullet^{* *}$ & Comet & चี \\
\hline$\cdot / T^{\mathrm{ns}}$ & $\cdot / Y^{n s}$ & $\mid \psi / \cdot \sigma^{\mathrm{ns}}$ & rT/ץq** & $-r / \lambda \omega^{\mathrm{ns}}$ & $r / \Delta)^{\text {ns }}$ & $-1 / \Delta \Lambda^{\mathrm{ns}}$ & $r / I q^{* *}$ & $r / q T^{*}$ & $r / \Delta q^{* *}$ & Shiralee & a \\
\hline$-\cdot / \cdot 1^{\mathrm{ns}}$ & $\cdot / 19^{n s}$ & $|\varepsilon / F|^{\mathrm{ns}}$ &.$- / \kappa^{e n s}$ & $1 \cdot / v q^{*}$ & $V / v \cdot{ }^{n s}$ & $-1 / F Y^{n s}$ & $-1 / 9 V^{* *}$ & $-r / r \cdot *$ & $-r / F)^{* *}$ & Kabel & \\
\hline$\cdot / \Lambda^{\mathrm{ns}}$ & $\cdot / \cdot e^{\mathrm{ns}}$ & $r / q)^{n s}$ & $q / \wedge q^{n s}$ & $1 \cdot / v \varepsilon^{*}$ & $\Lambda / q \Delta^{\mathrm{ns}}$ & $1 / r \Delta^{\mathrm{ns}}$ & $-\cdot / 0 \cdot * *$ & $-1 / \cdot \mu^{n s}$ & $-\kappa / \Delta \Lambda^{* *}$ & RGS003 & \\
\hline$\cdot|8|^{*}$ & $-\cdot / \cdot r^{\mathrm{ns}}$ & $r \cdot / 99^{* *}$ & $19 / 11^{*}$ & $\mathcal{F} / \mathrm{qV}^{\mathrm{ns}}$ & $r \cdot / r \cdot * *$ & $1 / \cdot \wedge^{\mathrm{ns}}$ & $-1 / \Delta q^{* *}$ & $1 / \Delta \Delta^{\mathrm{ns}}$ & - & دلكان & \\
\hline$-\cdot / / \Delta^{\mathrm{ns}}$ & $-/ / \omega^{*}$ & $-\Lambda / \Lambda V^{n s}$ & $-\varepsilon / 99^{\mathrm{ns}}$ & $r / / h^{\mathrm{ns}}$ & $N / I Y^{\mathrm{ns}}$ & $-\cdot 1 \cdot 1^{\mathrm{ns}}$ & $|/ F|^{* *}$ & $1 / \cdot \Delta^{\mathrm{ns}}$ & $\Gamma / / V^{* *}$ & Amica & \\
\hline$\cdot / \cdot r^{\mathrm{ns}}$ & $\cdot / / \mathrm{wns}^{\mathrm{ns}}$ & $-1 / \cdot 9^{\mathrm{ns}}$ & $11 / \kappa^{\mathrm{ens}}$ & $-\cdot / \Lambda \varepsilon^{n s}$ & $-r / \Delta \Delta^{\mathrm{ns}}$ & $\cdot / r \Delta^{\mathrm{ns}}$ & $. / 9)^{* *}$ & $-\cdot / v \Lambda^{\mathrm{ns}}$ & $\Gamma / / V^{* *}$ & Comet & $\overline{0}$ \\
\hline$-\cdot / / e^{\mathrm{ns}}$ & $-\cdot / / V^{\mathrm{ns}}$ & $-1 \Gamma / q . \mathrm{ns}$ & $-1 / / 9 r^{*}$ & $r / \Lambda . n s$ & $-\mid \kappa / \Lambda .^{* *}$ & $\cdot / r \Delta^{\mathrm{ns}}$ & $-\cdot 109^{* *}$ & $-1 / \kappa \Delta^{\mathrm{ns}}$ & $-1 / \Lambda r^{* * *}$ & Shiralee & ב \\
\hline$-\cdot / \cdot$ rns $^{\mathrm{ns}}$ &.$/ . \mathrm{ns}^{\mathrm{ns}}$ & $-\cdot / / r^{\mathrm{ns}}$ & $-r / v \Lambda^{\mathrm{ns}}$ & $-ه / \Delta \mu^{n s}$ & $-r / \mu \Lambda^{\mathrm{ns}}$ & $-\cdot 1 \cdot 1^{\mathrm{ns}}$ & $\cdot / \cdot \lambda^{\mathrm{ns}}$ & $-\cdot / V \Lambda^{\mathrm{ns}}$ & -1/rr*** & Kabel & \\
\hline \multirow[t]{2}{*}{$-\cdot / r q^{n s}$} & $\cdot / 4 \cdot *$ & $-\mathrm{N} \cdot \mathrm{r}^{\mathrm{ns}}$ & $-\cdot / 19^{\mathrm{ns}}$ & - & $-ه / \mathcal{F}^{\mathrm{ns}}$ & $-1 / \mathrm{F}^{\mathrm{ns}}$ & $-/ \Gamma \Delta^{\mathrm{ns}}$ &.$/ q^{n s}$ & $1 / \Delta \cdot * *$ & RGS003 & \\
\hline & .19 & N/ז & $V / \Delta V$ & $r / N r$ & r/qץ &.$/ 91$ &.$/ 19$ &.$/ 99$ & . & اشتباه معيار & \\
\hline
\end{tabular}

Table 3. Continued

ادامه جدول س

\begin{tabular}{|c|c|c|c|c|c|c|c|c|c|c|}
\hline \multicolumn{2}{|c|}{ شاخص برداشت } & \multicolumn{2}{|c|}{ عملكرد دانه } & \multicolumn{2}{|c|}{ ميزان روغن } & \multicolumn{2}{|c|}{ وزن هزار دانه } & \multirow{2}{*}{ دانه در غلاف } & \multirow{2}{*}{ لاينها } & \multirow{2}{*}{ تستر } \\
\hline غيرتنش & تنش & غيرتنش & تنش & غيرتنش & تنش & غيرتنش & تنش & & & \\
\hline$\cdot / V \varepsilon^{n s}$ & $T / F^{*} T^{*}$ & $-r V / M \Lambda^{n s}$ & $r / r v^{n s}$ & $-\cdot /\left.r\right|^{\mathrm{ns}}$ & $\cdot / 1 \cdot \mathrm{ns}$ &.$/\left.\cdot\right|^{\mathrm{cns}}$ & $\cdot / / \varepsilon^{\mathrm{ns}}$ & $1 / 4 \cdot \mathrm{ns}$ & دلغَان & \\
\hline$-r \mid \Lambda)^{*}$ & Ins & $-\Delta \Gamma q / \digamma \cdot * *$ & $\mid \varepsilon \Delta / V \Delta^{\mathrm{ns}}$ & $-\cdot / \Lambda \cdot n s$ & $\cdot \mid \Delta \xi^{n s}$ & $\cdot|r|^{* *}$ & $\cdot / \mathbb{4} \cdot *$ & $\cdot / 9 \Lambda^{\mathrm{ns}}$ & Amica & \\
\hline$\kappa / \wedge \omega^{* *}$ & $\cdot /\left.\right|^{\mathrm{ns}}$ & $\Gamma \cdot q / \Lambda . *$ & rrq/rrns & $-\cdot / r r^{n s}$ & & & -./. Hs & $\cdot / 9 . \mathrm{ns}$ & Comet & \\
\hline$-r / T \cdot n s$ & $-1 / \Delta \mu^{\mathrm{ns}}$ & $-I \Delta \Lambda / r \Lambda^{\mathrm{ns}}$ & $\begin{array}{c}1 \cdot 9 / \& \gamma^{\mathrm{ns}} \\
-\end{array}$ & $\cdot / v \cdot n s$ & & & $-\cdot / r \omega^{\mathrm{ns}}$ & $1 / 9 \Delta^{\mathrm{ns}}$ & Shiralee & ồ \\
\hline$-\cdot / \mathrm{V}^{\mathrm{ns}}$ & $-T / D)^{*}$ & TrI/VV* & -r../9r* & $\cdot \mid 99^{\mathrm{ns}}$ & ( & $\cdot 1 \cdot e^{\mathrm{ns}}$ & $-\cdot|r|^{\mathrm{ns}}$ & $-1 / \wedge \varepsilon^{n s}$ & Kabel & \\
\hline$\cdot / \Lambda^{\mathrm{ns}}$ & $\cdot / 1 \varepsilon^{\mathrm{ns}}$ & $\Lambda \mu / T v^{\mathrm{ns}}$ & N/I ws & $-\cdot / Y^{\mathrm{ns}}$ & $-\cdot / 11^{\mathrm{ns}}$ & $-/ r \omega^{* *}$ & $-\cdot / \cdot 9^{\mathrm{ns}}$ & $-r / \cdot \vartheta^{*}$ & RGS003 & \\
\hline$\cdot / 1 \cdot \mathrm{ns}^{-}$ & $-F / \Psi V^{* *}$ & $-V \subseteq \Delta / r \digamma^{* *}$ & DF\&/1.** & $\cdot / 9 \Delta^{\mathrm{ns}}$ & $-\cdot \mid q^{\mathrm{ns}}$ & $\cdot / r \Lambda^{* *}$ & $-\cdot / r \xi^{\mathrm{ns}}$ & -1/runs & $-\cdot / \Delta \xi^{n s}$ & \\
\hline$\cdot \mid q r^{\mathrm{ns}}$ &.$/ 91$ & $-q \cdot|f|^{\mathrm{ns}}$ & $M / \wedge \mathrm{rns}^{\mathrm{ns}}$ & $-\cdot 1 \cdot 1^{\mathrm{ns}}$ & (Wns & $\cdot / r r^{* *}$ & $\cdot / \mu^{\mathrm{ns}}$ & $-\cdot / \Delta \cdot \mathrm{ns}$ & $r / r)^{*}$ & \\
\hline$-\cdot(\Delta)^{\mathrm{ns}}$ & $\cdot / 19^{\mathrm{ns}}$ & $-|V Q /| T^{\mathrm{ns}}$ & $-1 / V \cdot n s$ & $\cdot / \wedge r^{\mathrm{ns}}$ & - & $\cdot / \cdot \wedge^{\mathrm{ns}}$ & $\cdot 1 \cdot \mathrm{cns}^{\mathrm{ns}}$ & $-|/ T|^{\mathrm{ns}}$ & Comet & $\cdot / 11^{\mathrm{ns}}$ \\
\hline . $/$ arns & T/r* & 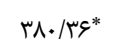 & $r \cdot N / r V^{n s}$ & $-\cdot / \mathrm{A}^{\mathrm{ns}}$ & $-1 /$ rns & . & $\cdot / / r^{\mathrm{ns}}$ & $\cdot \mid \Delta)^{\mathrm{ens}}$ & Shiralee & ./I" \\
\hline$-\cdot / 9 \Delta^{\mathrm{ns}}$ & $1 / V^{n s}$ & $\mid \Delta V / V \xi^{n s}$ & ISV/A $Y^{n s}$ & $-\cdot / q \mu^{\mathrm{ns}}$ & $\cdot(f)^{\mathrm{ns}}$ & | & $-\cdot / \cdot r^{n s}$ & $r / \cdot \mathrm{e}^{\mathrm{ns}}$ & Kabel & \\
\hline 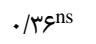 & $-1 / \uparrow \Lambda^{\mathrm{ns}}$ & FET/Vq** & $10 T / V V^{n s}$ & $\cdot 1 \cdot c^{\mathrm{ns}}$ & $\cdot / / \mathrm{V}^{\mathrm{ns}}$ & $\cdot 1 \cdot \mathrm{V}^{\mathrm{ns}}$ & $-\cdot / \cdot \Delta^{\mathrm{ns}}$ & . & RGS003 & \\
\hline$-\cdot / \wedge^{\mathrm{ns}}$ & 1/৭ & Vqr/Dr** & $\Delta F^{F} / / \Lambda r^{* *}$ & $-\cdot / V^{\mathrm{ns}}$ & $\cdot / \Delta Y^{n s}$ & $-(\mid)^{* *}$ & $\cdot 1 \cdot 9^{\mathrm{ns}}$ & $-\cdot 1 \cdot 9^{\mathrm{ns}}$ & دلعان & \\
\hline$r / r \cdot \mathrm{ns}$ & - & ๑^৭/^.* & $\begin{array}{c}\mid \Lambda F / \Delta q^{\mathrm{ns}} \\
-\end{array}$ & $\cdot / \wedge \Upsilon^{n s}$ & $-1 / \Lambda \cdot n s$ & - & $-\cdot|\Delta|^{* *}$ & $-\cdot / \uparrow \lambda^{\mathrm{ns}}$ & Amica & \\
\hline$-\boldsymbol{r} / \boldsymbol{\mu} \cdot * *$ & $-1 / \cdot e^{\mathrm{ens}}$ & $-\mid \mu r / \& V^{n s}$ & $\begin{array}{c}r T V / \Delta H^{n s} \\
-\end{array}$ & $-\cdot / 9 \cdot \mathrm{ns}$ & $\cdot / \Delta r^{n s}$ & $-\cdot / 1 \mathrm{v}^{* * *}$ & $-\cdot / \cdot r^{\mathrm{ns}}$ & $\cdot|\rangle^{\mathrm{ns}}$ & Comet & D \\
\hline $1 / q^{n s}$ & $-\cdot / \Lambda r^{n s}$ & $-r T r / \cdot \Lambda^{n s}$ & $\begin{array}{c}1 \cdot 1 / 9)^{\mathrm{ns}} \\
-\end{array}$ & $\cdot / 19^{\mathrm{ns}}$ & $-1 / . . n s$ & $\cdot / F \theta^{* *}$ & $\cdot / 11^{\mathrm{ns}}$ & $-r / 19^{n s}$ & Shiralee & $\stackrel{0}{\beth}$ \\
\hline $1 / V r^{n s}$ & $\cdot / \Lambda \cdot^{\mathrm{ns}}$ & $-F \vee q / \Delta \mu^{* * *}$ & $\mid r m / \cdot q^{n s}$ & $\cdot / r \Lambda^{\mathrm{ns}}$ & $1 / \wedge r^{n s}$ & $\cdot / q^{* *}$ & $\cdot\left(\left.r\right|^{\mathrm{ns}}\right.$ & $-\cdot / 19^{\mathrm{ns}}$ & Kabel & \\
\hline$-\cdot|\omega|^{\mathrm{ns}}$ & $1 /$ rens & $-\Delta \uparrow \& / \cdot \mu^{* * *}$ & $\begin{array}{c}|9 \cdot / 9|^{\mathrm{ns}} \\
-\end{array}$ & $\cdot 1 \cdot \varepsilon^{\mathrm{ns}}$ & $-\cdot / \cdot \mathrm{V}^{\mathrm{ns}}$ &.$/ 19^{* *}$ & $\cdot / \mathrm{rns}^{\mathrm{ns}}$ & $r / \varphi . n s$ & RGS003 & \\
\hline $1 / K T^{e}$ & $1 / \cdot 1$ & $\mid f \Psi / \Lambda \varepsilon$ & $1 F q / \Delta \Lambda$ & $\cdot / \Delta T$ & $1 / .9$ & .1 .9 &.$/ 1 \Lambda$ & $1 / T V$ & اشتباه معيار & \\
\hline
\end{tabular}


جدول ع أ برآوردهاى ميزان هتروزيس (\%) براى صفات مختلف زنوتيڤهاى كلزا در شرايط تنش و عدم تنش رطوبتى (\%) Table 4. Estimates of heterosis (\%) for different traits in rapeseed genotypes under drought stress and non stress

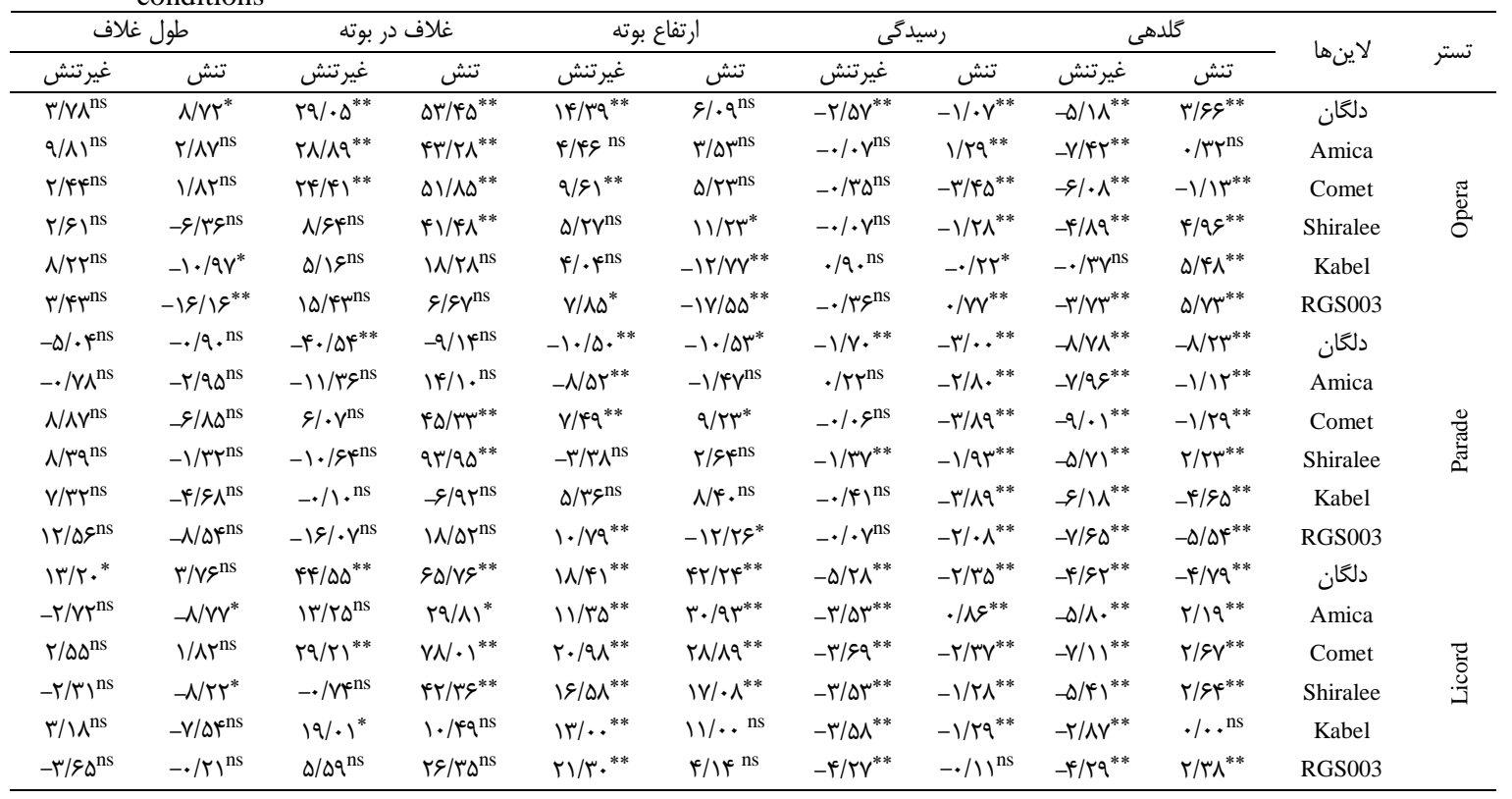

Table 4. Continued

ادامه جدول f

\begin{tabular}{|c|c|c|c|c|c|c|c|c|c|c|}
\hline \multicolumn{2}{|c|}{ شاخص برداشت } & \multicolumn{2}{|c|}{ عملكرد دانه } & \multicolumn{2}{|c|}{ ميزان روغن } & \multicolumn{2}{|c|}{ وزن هزار دانه } & \multirow{2}{*}{ دانه در غاف } & \multirow{2}{*}{ لاينها } & \multirow{2}{*}{ تستر } \\
\hline غيرتنش & تنش & غيرتنش & تنش & غيرتنش & تنش & غيرتنش & تنش & & & \\
\hline $1.1 .9^{\mathrm{ns}}$ & $\Gamma \cdot|\Lambda|^{* *}$ & $\Delta \Psi / q^{* *}$ & QQ/घ9** & $1 / v^{n s}$ & $r / V r^{n s}$ & $r \cdot \mid \Delta f^{* * *}$ & $|\varphi /| \psi|*|^{* *}$ & $-\Gamma / A \gamma^{n s}$ & دلكان & \\
\hline$\cdot / T Y^{n s}$ & $r T / V \Lambda^{* *}$ & $\Lambda \Psi / \Delta)^{* *}$ & $\Delta \mathrm{V} / \cdot \mathrm{V}^{* *}$ & $.1 \cdot 9^{\mathrm{ns}}$ & $\Delta / q e^{\mathrm{ns}}$ & $r N / V I^{* * *}$ & $F \mid / \Delta \varphi^{* *}$ & $-\mid V / \varphi^{\mathrm{ns}}$ & Amica & \\
\hline$q \psi / . \varphi^{* * *}$ & $r V / /$ F** $^{* *}$ & $\| \Delta / \Delta)^{* * *}$ & $\mid \mathcal{E N / r q * *}$ & $r / q e^{* * *}$ & $r / \Lambda q^{n s}$ & $\mid \mathrm{V} / \mathrm{T} \cdot{ }^{* * *}$ & $r y / \cdot r^{*}$ & $\cdot / r \mathrm{~V}^{\mathrm{ns}}$ & Comet & $\approx$ \\
\hline$-\mid \kappa / \Delta \varepsilon^{\mathrm{ns}}$ & r & $F V / l e^{* * *}$ & $\Delta F / \cdot 1^{* *}$ & $s / r \omega^{* *}$ & $|\Gamma / 9|^{* *}$ & $r r / r q^{* *}$ & $r \Delta / \varphi^{* *}$ & $-11 / \Delta V^{n s}$ & Shiralee & के \\
\hline$-r / v \cdot n s$ & $\mid \varepsilon / \mathrm{Q}^{* *}$ & $\checkmark \cdot / \omega \cdot{ }^{* *}$ & $\kappa \psi / F T^{*}$ & $\kappa / \Delta F^{* * *}$ & $r / V^{m \text { ns }}$ & $|V / F|^{* *}$ & $\widetilde{V} / \& \omega^{* *}$ & $-r \Psi / \Delta \varphi^{* * *}$ & Kabel & \\
\hline$r \Delta / v \varphi^{* *}$ & $r r / \cdot r^{* *}$ & १५/\&८** & $\Gamma \Delta / / Q^{* *}$ & $\cdot / \Lambda \omega^{\mathrm{nS}}$ & $\mathrm{f} / \mathrm{V} \cdot \mathrm{ns}$ & $\mid V / \Lambda e^{* *}$ & $F V / M r^{* *}$ & - & RGS003 & \\
\hline$\Delta / \Delta \cdot{ }^{n s}$ & $19 / \cdot 9^{* *}$ & $r / \Delta V^{n s}$ & $|1 / \wedge|^{\mathrm{ns}}$ & $g(\Delta)^{* *}$ & $r / r \lambda^{\mathrm{ns}}$ & $\kappa * / V \Lambda^{* *}$ & $N / \Delta F^{*}$ & $r / \mu)^{n s}$ & دلرًان & \\
\hline $\mid V / \& 9^{*}$ & $r q / \mu^{* * *}$ & $\Lambda \mathrm{V} / \Lambda Q^{* *}$ & $1 \cdot / r^{n s}$ & $r / \cdot \mu^{*}$ & $V / V e^{*}$ & $\mathrm{rV} / \cdot \wedge^{* * *}$ & १/. $\wedge^{\mathrm{ns}}$ & $-9 / v \cdot \mathrm{ns}$ & Amica & \\
\hline$r q / r \Lambda^{* *}$ & $\Delta q / \mu \mu^{* * *}$ & $99 / 19^{* *}$ & $|r q / /|^{* * *}$ & $9 / 1^{* *}$ & $r / \psi^{n s}$ & \} / \wedge 9 ^ { * * } $&{g / V^{e n s}} &{\text { N/qV }} &{\text { Comet }} &{\approx} \\
{\hline-r / r q^{n s}} &{\Delta F / \Lambda 1^{* *}} &{\Delta F / \& \Lambda^{* *}} &{\Delta \cdot / T \cdot * *} &{r / v \cdot * *} &{\Delta / \cdot q^{\mathrm{ns}}} &{r \mid / r^{* * *}} &{r r / F)^{* *}} &{1 / . \mathrm{ns}^{\mathrm{ns}}} &{\text { Shiralee }} &{\text { ¿ }} \\
{\hline-r / r \cdot{ }^{n s}} &{\kappa q / \cdot r^{* * *}} &{\text { r }} &{\Delta \Gamma / T V^{* *}} &{r / A r^{n s}} &{11 / r \Lambda^{* *}} &{\text { ᄉ/q.** }} &{T V / \& q^{* *}} &{\text { r/А } \mu^{n s}} &{\text { Kabel }} &{ } \\
{\hline r \mu / r . * *} &{r q / \Delta \Delta^{* *}} &{91 / \& \varphi^{* * *}} &{19 / 4 .{ }^{\mathrm{ns}}} &{r / \mu)^{*}} &{\Delta / \Delta \Lambda^{\mathrm{ns}}} &{\text { r }} &{\text { 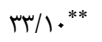 }} &{\mid r / \Lambda \cdot{ }^{n s}} &{\text { RGS003 }} &{ } \\
{\hline-\mid V / A r^{*}} &{\text { Q } 1 / \cdot 1^{* * *}} &{q \varepsilon / \mu \Gamma^{* * *}} &{\wedge \varepsilon / \wedge \varepsilon^{* *}} &{1 / 9 r^{n s}} &{r / 9 . n s} &{\text { r }} &{r N / Y \Lambda^{* *}} &{11 / q^{\mathrm{ns}}} &{\text { دلگان }} &{ } \\
{\hline 1 / 1 n^{\mathrm{ns}}} &{\text { M/A.** }} &{\mid \mathrm{V} / / \mathrm{Q}^{* *}} &{r \mu / Q)^{n s}} &{\Delta / r \Lambda^{* *}} &{-r / 9 . \mathrm{ns}} &{1 . / F V^{* *}} &{-ه / q \mathrm{Hns}^{\mathrm{ns}}} &{-\varepsilon / q^{\mathrm{ns}}} &{\text { Amica }} &{ } \\
{\hline-11 / \mathcal{M}^{\mathrm{ns}}} &{\Delta \mathrm{N} / \cdot \mathrm{r}^{* * *}} &{\text { १९/ศน** }} &{\mid \mu F / V^{* *}} &{\Delta / 1 . * *} &{r / r{ }^{n s}} &{\mid Q / \backslash \Lambda^{* *}} &{-\mid Q / \Psi r^{n s}} &{W / V \Delta^{*}} &{\text { Comet }} &{\bar{g}} \\
{\hline-\mid \varepsilon / g^{\mu *}} &{\boldsymbol{F} \mathcal{N} / \boldsymbol{F}^{\mathcal{*}} * *} &{\Gamma q / \wedge \mathcal{F}^{* *}} &{\Delta T / \mathcal{L U}^{* * *}} &{8 / 0 \cdot * *} &{r / 4 . n s} &{\Delta \Gamma / F V^{* *}} &{\text { MI/ }} &{-1 \cdot / r v^{\mathrm{ns}}} &{\text { Shiralee }} &{\stackrel{0}{3}} \\
{\hline-\mid r / T V^{\mathrm{ns}}} &{\Delta T / \cdot \wedge^{* *}} &{\text { TN/TT* }} &{\wedge) / \Delta V^{* *}} &{\Delta / . .^{* *}} &{11 / / Q^{* *}} &{\text { rه/द.*** }} &{\Gamma \Delta / r^{* * *}} &{-\Delta / / \omega^{\mathrm{nS}}} &{\text { Kabel }} &{ } \\
{\hline-r / q v^{n s}} &{\text { |q/g }} &{\varepsilon \Delta / \mu^{* * * *}} &{r / / r^{n s}} &{r / q^{\mathrm{rns}^{\mathrm{ns}}}} &{1 / \mathrm{v}^{\mathrm{ns}}} &{\mid \kappa+/ \& q^{* *}} &{F V / V r^{* *}} &{\text { TN/D.** }} &{\text { RGS003 }} &{ } \\
$\hline
\end{tabular}


غيرتنش بهترين بودند. با توجه به نتايج تركيبذيذيرى (SA)

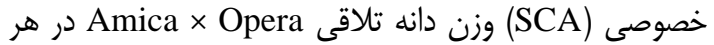

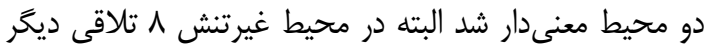

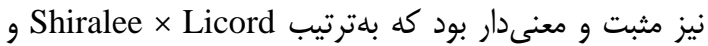
Shiralee × Parade

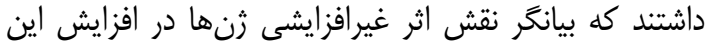

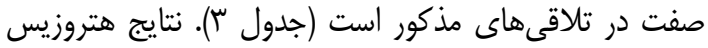

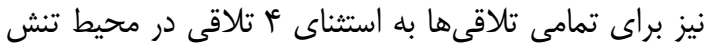

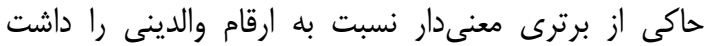

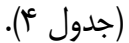

ميزان روغن و عملكرد دانه

بيشترين GCA مثبت و معنى دان دار هر دو محيط براى صن صفت

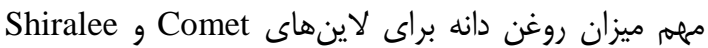

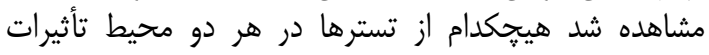

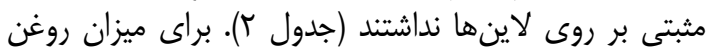

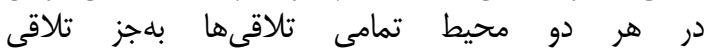
Shiralee × Opera

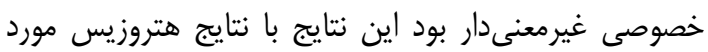

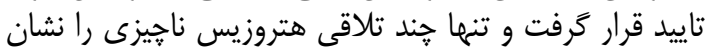

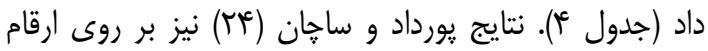

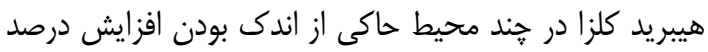
روغن به علت هتروزيس بودا.

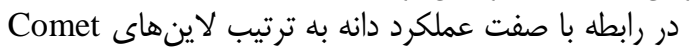

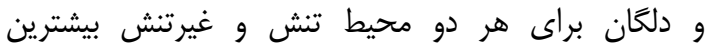

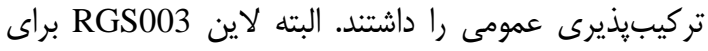

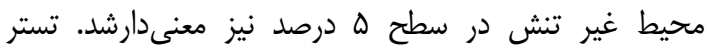

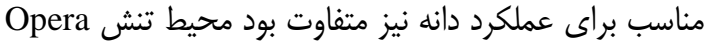

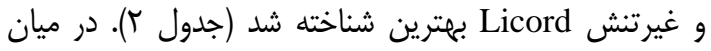

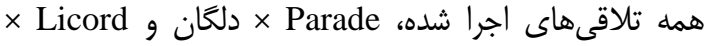

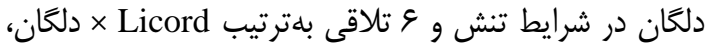
$\times$ Parade ،Amica $\times$ Licord RGS003 × Parade Comet × Opera و Kabel × Opera ،Shiralee عدمتنش داراى تركيبيذيرى خصوصى مثبت و معنى منى إدار از

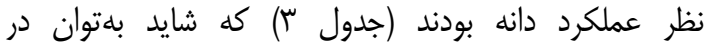

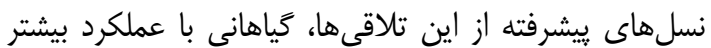

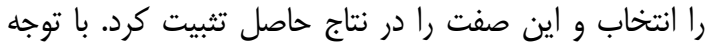

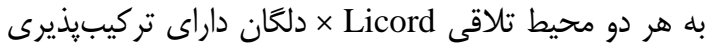

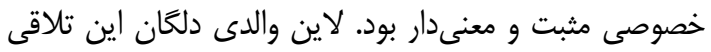

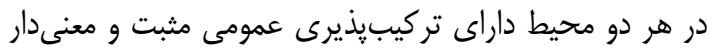

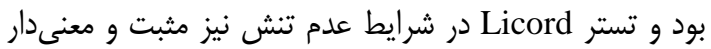

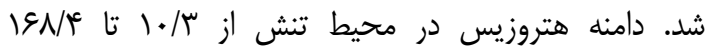
IVI/T و محيط غيرتنش از (Comet × Opera) (Amica × Licord)

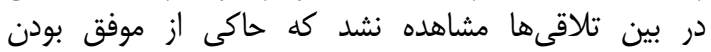

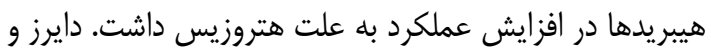

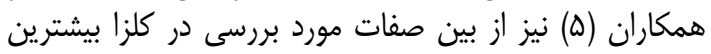

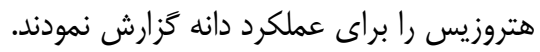
شاخص برداشت

در نهايت براى شاخص برداشت، لاينها و تسترهاى برتر

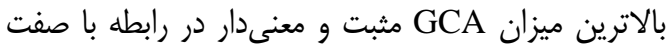

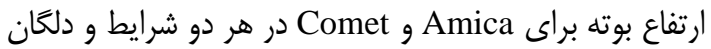

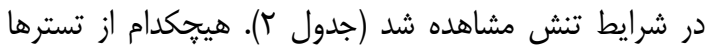

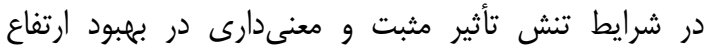

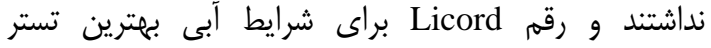

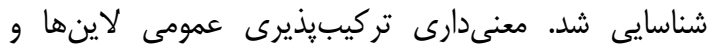

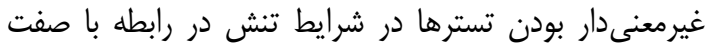

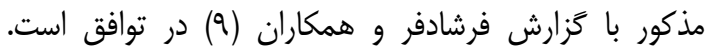

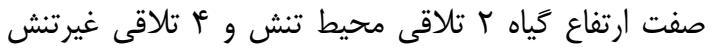

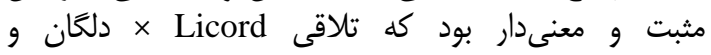

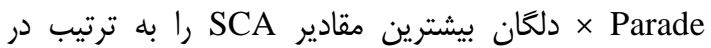
محيط تنش و غيرتنش را داشتند (جدول دار). صفت ارتفاع بوته

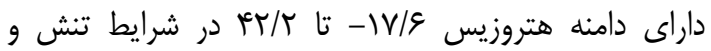

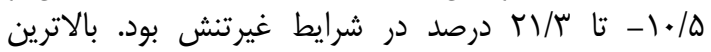

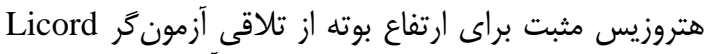

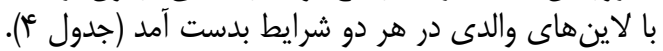

اجزاى عملكرد - الاين

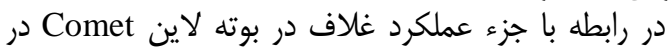
هر دو شرايط و لاين دلكان در محيط تنش و و تسترهاى درائ

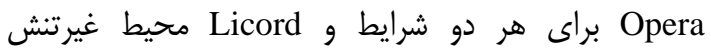
بيشترين GCA مثبت و معنى دار را به خود اختصاص داص دادند (جدول r). هيبريد Shiralee × Parade داراى بيشترين

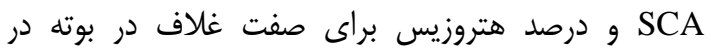
شرايط تنش و هيبريد Licord × دائ دلكان در هر دو دو محيط

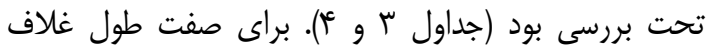

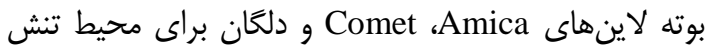

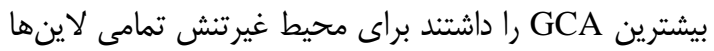

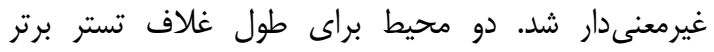

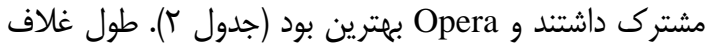

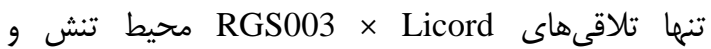
هicord

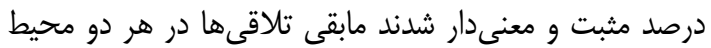

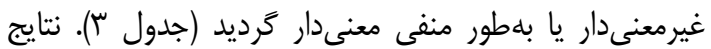

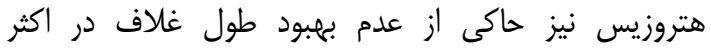
تلاقىها در دو محيط را داشت. تلاقى رقم

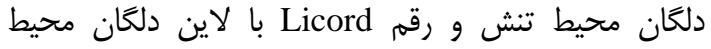
غيرتنش داراى هتروزيس مثبت و معنى دارى دار در سطح احتمال

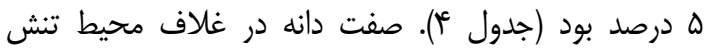

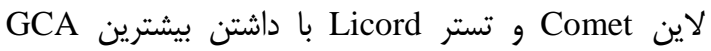

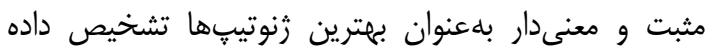

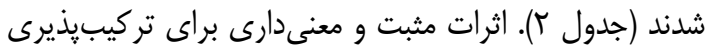

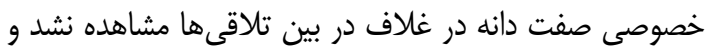
همجنين تنها تلاقى رقم Licord بان دالاين RGS003 و Comet

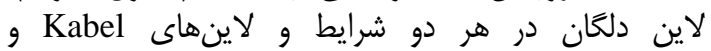
RGS003 تنها براى محيط تنش بيشترين GCA معند

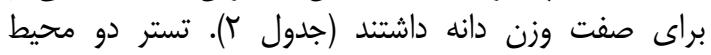
متفاوت بود و Opera در محيط تنش و Licord در محيط دان محيط 
صفت عملكرد دانه در تلاقى بين لاينهاى زمستانه با بهاره،

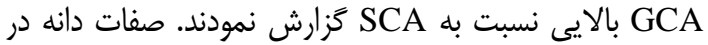

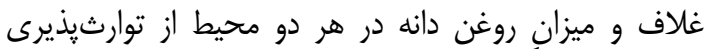

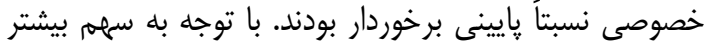

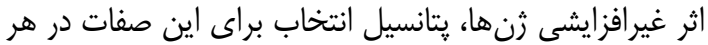

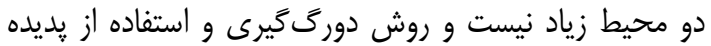

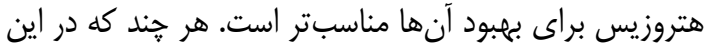

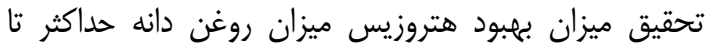

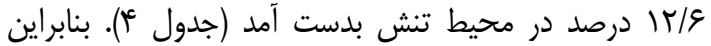

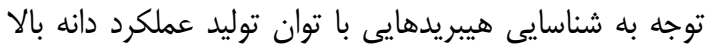

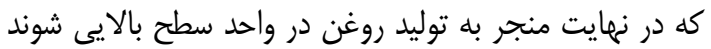

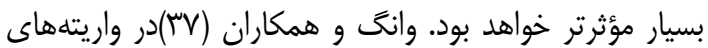

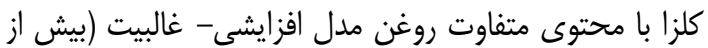

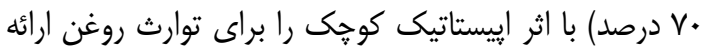

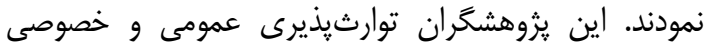

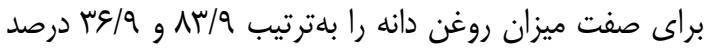

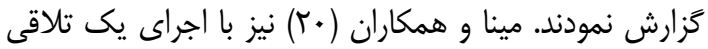

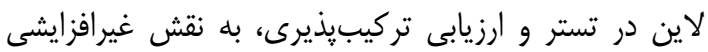

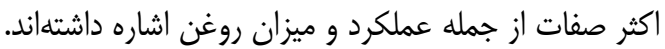

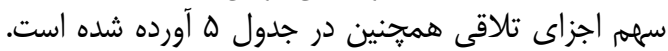

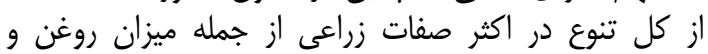

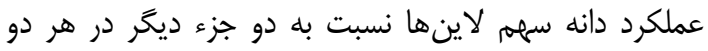

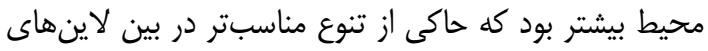

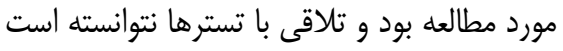

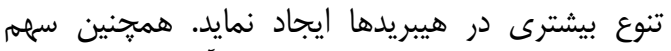

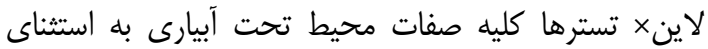

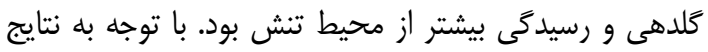

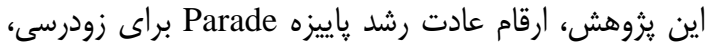

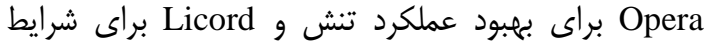

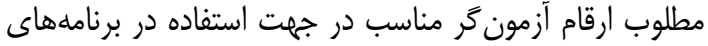

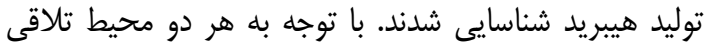
الشت ×icord

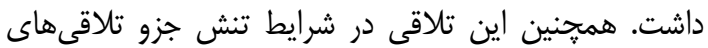

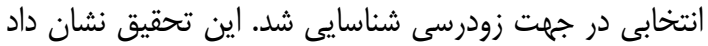

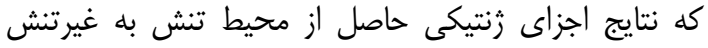

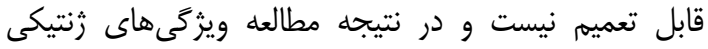

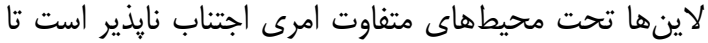
بر آورد عمل زنها با با دقت بيشترى همراه باشد.

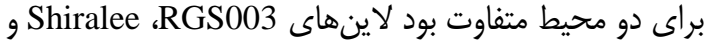

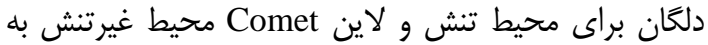

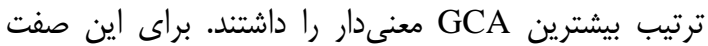

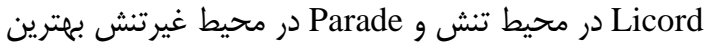

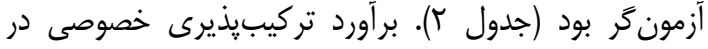

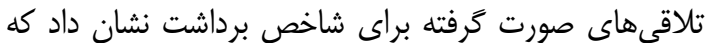

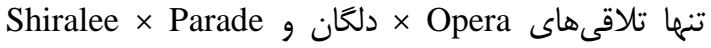

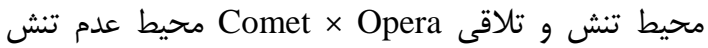

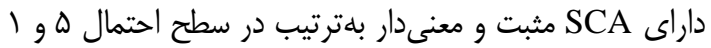

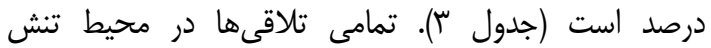

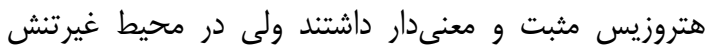

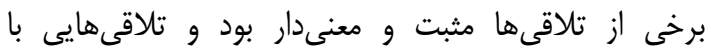

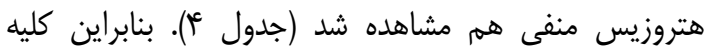

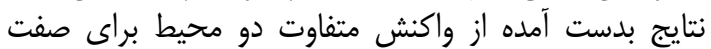
مزبور را نشان داد. يار امتر هاى زينتيكى إنى محاسبه اجزاى واريانس زنتيكى (واريانس افزايشى، غالبيتيت

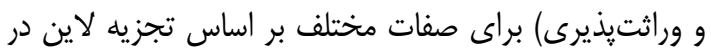

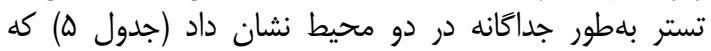

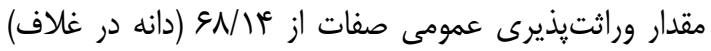

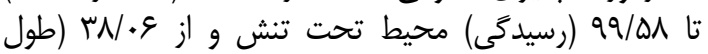

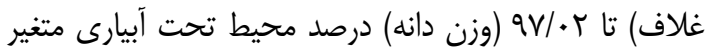

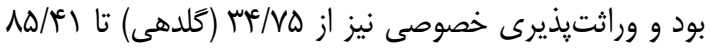

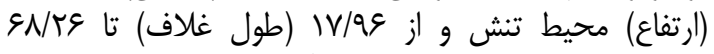

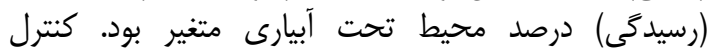

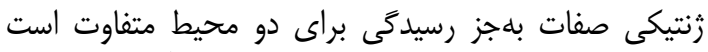

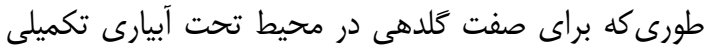

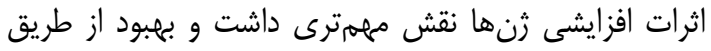

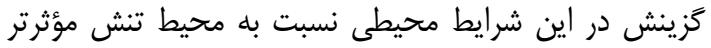

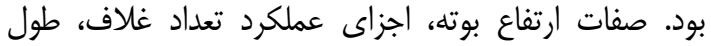

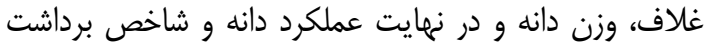

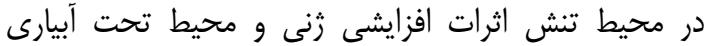

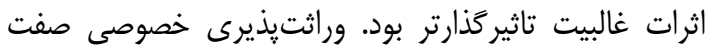

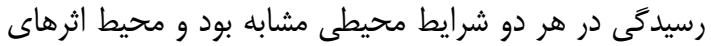

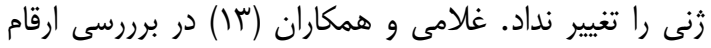

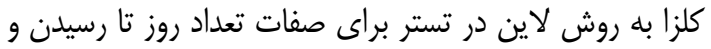

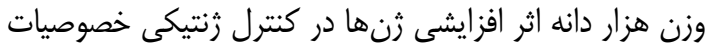

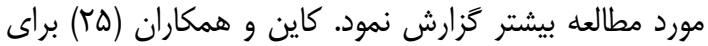


جدول ه- برآوردهاى اجزاى واريانس، وراثتيذيرى و سهم لاين، تستر و اثرمتقابل براى صفات زراعى زنوتيڤهاى كلزا در شرايط تنش و عدم تنش رطوبتى

Table 5. Estimates of variance components, heritability and contribution of the line, tester, and interaction for agronomic traits of rapeseed genotypes under drought stress and non stress conditions

\begin{tabular}{|c|c|c|c|c|c|c|c|c|c|c|c|}
\hline \multicolumn{2}{|c|}{ طول غلاف } & \multicolumn{2}{|c|}{ غلاف در بوته } & \multicolumn{2}{|c|}{ ارتفاع بوته } & \multicolumn{2}{|c|}{ رسيدگى } & \multicolumn{2}{|c|}{ كلدهى } & \multirow{2}{*}{\multicolumn{2}{|c|}{ اجزاى زنتيكى }} \\
\hline غيرتنش & تنش & غيرتنش & تنش & غيرتنش & تنش & غيرتنش & تنش & غيرتنش & تنش & & \\
\hline$\cdot 1 \cdot 4^{\mathrm{f}}$ & תז/. & $19 \Delta / 9 \Delta$ & १\&स/1. & $V Q / F V$ & $1.9 \Delta / V T$ & $\Delta / V V$ & $V / 99$ & $1 . / V 9$ & $\mid \pi / 99$ & افزايشى & \\
\hline$\cdot 1 \cdot \Delta$ & $\cdot / \cdot v$ & . & qو/شس & $\Lambda \Delta / 1 \varphi$ & هץ/N" & $1 / \cdot e^{c}$ & $r / q \Lambda$ & זس/ץ & r & غالبيت & \\
\hline rN/.G & $\Lambda \Delta / / F$ & $\Lambda \cdot / r \mid$ & $9) / 11$ & $\Lambda T / \Delta S$ & $q \& / T$. & $\Lambda \cdot / \Delta H$ & १৭/०१ & $\Lambda V / \Lambda F$ & ११/५ & عمومى & وراثتيذيرى \\
\hline $1 \mathrm{~V} / \mathrm{ag}$ & GQ/ & rq/1r & GN/TF & rN/vq & $\left.\Lambda \Delta /{ }^{c}\right\rangle$ & SN/TG & 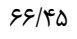 & $S V / 1 D$ & $M F / V D$ & خصوصى & (درصد) \\
\hline$r . / 11$ & $94 \%$ & Tr/TV & سו/ & $r r / .$. & $M N / \Delta S$ & $\Delta S / F \Delta$ & TN/FT & $\mathrm{rq} / \Delta q$ & $19 / \Delta F$ & لاين & \\
\hline rT/qr & $19 / 1 F$ & rV/gr & rו/ & Tr/Tr & $q / 9 V$ & $\mid f / / f$ & $\mid s / 1$. & גT/Tג & Tr/IF & لاين× تستر & \\
\hline
\end{tabular}

Table 5. Continued

ادامه جدول ه

\begin{tabular}{|c|c|c|c|c|c|c|c|c|c|c|}
\hline \multicolumn{2}{|c|}{ شاخص برداشت } & \multicolumn{2}{|c|}{ عملكرد دانه } & \multicolumn{2}{|c|}{ ميزان روغن دانه } & \multicolumn{2}{|c|}{ وزن هزار دانه } & \multirow{2}{*}{ دانه در غلاف } & \multirow{2}{*}{\multicolumn{2}{|c|}{ اجزاى زنتيكى }} \\
\hline غيرتنش & تنش & غيرتنش & تنش & غيرتنش & تنش & غيرتنش & تنش & & & \\
\hline$\Delta / \Delta \cdot$ & $14 / 94$ & TVN\&V./QD & $|\nvdash \wedge \mu \cdot 1 / \wedge|$ & $\cdot / V \cdot$ & ( &.$/ 14$ & $\cdot / \Delta \Delta$ & $\Delta / 9 \mu$ & افزايشى & \\
\hline$\Delta / \& \Delta$ & r/ar & $r M \cdot \Lambda I / F \Delta$ & $\mid V T I T / q T$ & ./ & $1 / \cdot 9$ &.$/ 11$ & $.1 \cdot \mathrm{r}^{\mathrm{C}}$ & $T / \cdot G$ & غالبيت & \\
\hline VN/IV & 19/DT & $94 / 19$ & $q \mu / T F$ & sq/VV & $R \cdot /$ et & $q Y / \cdot r$ & $9 . / 1 r$ & SN/IF & عمومى & وراثتيذيرى \\
\hline$r q / r \Delta$ & MV/M & $r \Delta / A r$ & $\Lambda r / \Delta r$ & er/qu & $\Delta S / T \Delta$ & $01 / V 1$ & $\Lambda \mu / \mu$ & $\Delta \cdot / \Delta \Delta$ & خصوصى & (درصد) \\
\hline$r q / \Delta r$ & $v e / r$. & DI/FT & سז/או & $\Delta F / \backslash \Delta$ & $q q / r \Delta$ & $\Delta q / V \Delta$ & 1)/98 & $9 \cdot 119$ & لاين & \\
\hline$r r / \Delta V$ & $r / v \wedge$ & N/gr & $ه / 91$ & १/९८ & $\Delta / \cdot \Gamma^{C}$ & $r / F \Lambda$ & $q / \uparrow \wedge$ & $1 . / 97$ & تستر & سקו (נ) \\
\hline " & rI/9r & rq/qq & Q/V६ & . & $r \Delta / r)$ & rv/ve & $N / \Delta S$ & $r N / q$. & لاين× تستر & \\
\hline
\end{tabular}

1. Arefi, S., A. Nabipoor and H. Samizade. 2015. Evaluation of combining ability of sunflower lines based on line $\times$ tester analysis under water stress and non-stress conditions. Journal of Crop Breeding, 7 (15): 115-125 (In Persian).

2. Butruille, D.V., R.P. Guries and T.C. Osborn. 1999. Increasing yield of spring oilseed rape Hybrids through introgression of winter germplasm. Crop Science, 39: 1491-1496.

3. Choukan, R., A. Estakhr, A. Afarinesh, Gh.R. Afsharmanesh, M.R. Shiri, A. Mosavat and Sh. Fareghei.2014. Combining ability of tropical maize lines derived from CIMMYT germplasm in crossing with temperate lines. Iranian Journal of Crop Sciences, 16(4): 334-345 (In Persian).

4. DelaVega, A.J. and S.C. Chapman. 2006. Multivariate analyses to display interactions between environment and general or specific combining ability in hybrid crops. Crop Science, 46: 957-967.

5. Diers, B.W., P.B.E. McVetty and T.C. Osborn. 1996. Relationship between heterosis and genetic distance based on restriction fragment length polymorphism markers in oilseed rape (Brassica napus L.,). Crop Science, 36: 79-83.

6. Engqvist, G.M. and H.C. Becker. 1991. Heterosis and epistasis in rapeseed estimated from generation means. Euphytica, 58: 31-41.

7. Fan, X.M., X.F. Yin, Y.D. Zhang, Y.Q. Bi, L. Liu, H.M. Chen and M.S. Kang. 2016. Combining ability estimation for grain yield of maize exotic germplasm using testers from three heterotic groups. Crop Science, 56: 2527-2535.

8. FAO. 2016. Food and agriculture organization of the United Nations. FAOSTAT statistics database. Available online at: http://www.faostat.fao.org.

9. Farshadfar, E., Z. Kazemi and A.Yaghotipoor. 2013. Estimation of combining ability and gene action for agro-morphological characters of rapeseed (Brassica napus L.) using linextester mating design. International Journal of Advanced Biological and Biomedical Research 1: 711-717.

10. Fehr, W.R. 1993. Principles of cultivar development. MacMillan Publ. Co. New York, USA, 1, 342 pp.

11. Gami, R.A. and R.M. Chauhan. 2013. Heterosis and combining ability analysis for seed yield and its attributes in Indian mustard [Brassica juncea (L.) Czern \& Coss.]. Indian Journal of Agricultural Research, 47: 535-539.

12. Ghaffari, M., F. Shariati and A. Lack. 2016. Genetic analysis of seed and oil yield related agronomic traits under optimum and limited irrigation in sunflower. Iranian Journal of Dryland Agriculture, 5(1): 225-247 (In Persian).

13. Gholami, H., M. Moghaddam and V. Rameeh. 2008. Estimation of combining ability in rapeseed (Brassica napus L.) using line $\times$ tester cross method. Seed and Plant Improvement Journal, 24(3): 399-411 (In Persian). 
14. Ghorbani, H.R., H. Samizadeh Lahiji, B. Rabiei and M. Allah Gholipour. 2014. Line $\times$ tester analysis for yield and yield components in rice lines. Iranian Journal of Field Crop Science, 44(4): 683-692 (In Persian).

15. Hale, A.L., M.W. Farnham, M.N. Nzaramba and C.A. Kimbeng. 2007. Heterosis for horticultural traits in Broccoli. Theoretical Applied Genetics, 115: 351-360.

16. Kanatti, A., K. Nath Rai, K. Radhika and M. Govindaraj. 2016. Tester effect on combining ability and its relationship with line performance per se for grain iron and zinc densities in pearl millet. Crop Science, 56: 689-696.

17. Kemptorn, O. 1957. An introduction to genetic statistics. John Wiley and Nordskoy. Inc. London, Champman and Hall, LTD.

18. Kothari, N., B.T. Campbell, J.K. Dever and L.L. Hinze. 2016. Combining ability and performance of cotton germplasm with diverse seed oil content. Crop Science, 56: 19-29.

19. Kumar, M., P.K. Singh and N.P. Singh 2000. Line $\times$ tester analysis for seed yield and its components in linseed (Linum usitatissimum L.). Annals of Agricultural Research, 21(4): 485-489.

20. Meena, H.S. , A. Kumar, B. Ram, V.V. Singh, P.D. Meena, B.K. Singh and D. Singh. 2015. Combining ability and heterosis for seed yield and its components in Indian mustard (Brassica juncea L.). Journal of Agricultural Science and Technology, 17: 1861-1871.

21. Moghaddam, M. and H. Amiri Oghan. 2010. Biometrical methods in quantitative genetic analysis. Publication of Tabriz University, Tabriz, Iran. 415 pp (In Persian).

22. Narro, L., S. Pandey, J. Crossa, C. De Leo'n and F. Salazar. 2003. Using line - tester interaction for the formation of yellow maize synthetics tolerant to acid soils. Crop Science, 43: 1718-1728.

23. Ofori, A., and H.C. Becker. 2008. Breeding of brassica Rapa for biogas production: heterosis and combining ability of biomass yield. Bioenergy Research, 1: 98-104.

24. Pourdad, S. S. and J.N. Sachan. 2003. Study On heterosis and inbreeding depression in agronomic and oil quality characters of rapeseed (Brassica napus L.). Seed and Plant Improvement Journal, 19(3): 413-427 (In Persian).

25. Qian, W., O. Sass, J. Meng, M. Li, M. Fruen and C. Jung. 2007. Heterotic patterns in rapeseed (Brassica napus L.): I. crosses between spring and Chinese semi-winter lines. Theoretical Applied Genetics, 115: 27-34.

26. Quijada, P.A., J.A. Udall, H. Polewicz, R. Vogelzang and T.C. Osborn. 2004. Phenotypic effects of introgressing French winter germplasm into hybrid spring canola. Crop Science, 44: 1982-1989.

27. Rahman, H. and B. Kebede. 2012. Improvement of spring canola Brassica napus (L.) by use of winter canola. Journal Oilseed Brassica, 3: 1-17.

28. Rahman, H., A. Bennett and R.C. Yang. 2016. Patterns of heterosis in three distinct inbred populations of spring Brassica napus Canola. Crop Science, 56: 2536-2545.

29. Rajcan, I., K.J. Kasha, L.S. Kott and W.D. Beverdorf. 1999. Detection of molecular markers associated with linolenic and erucic acid levels in spring rapeseed (Brassica napus L.). Euphytica, 105: 173-181.

30. Rameeh V.O. 2009. Estimation of heritability and heterosis for agronomic traits and oil content in rapeseed spring varieties. Journal of Crop Breeding, 1(4): 1-13 (In Persian).

31. Reif, J.C., Y. Zhao, T. Würschum, M. Gowda and V. Hahn. 2013. Genomic prediction of sunflower hybrid performance. Plant Breeding, 132: 107-114.

32. Shehzad, A., H.A. Sadaqat, M. Ali and M.F. Ashraf. 2015. Combining ability analysis and geneticeffects studies for some important quality characters in Brassica napus L. Turkish Journal of Agriculture - Food Science and Technology, 3(10): 790-795.

33. Singh, R.K. and B.D. Chaudhary.1977. Biometrical methos in quantitative genetic analysis. Kalyani Pub., New Delhi.

34. Starmer, K.P., J. Brown, and J.B. Davis. 1998. Heterosis in spring canola hybrids grown in northern Idaho. Crop Science, 38: 376-380.

35. Teklewold, A. and H.C. Becker. 2005. Heterosis and combining ability in a diallel cross of Ethiopian mustard inbred lines. Crop Science, 45: 2629-2635.

36. Wang, J.S., X.F. Wang, Y.F. Zhang, Z. Zhang, J.H. Tian and D.R. Li. 2007. Study on heterosis among subspecies or varieties in B. campestris L. 12th International Rapeseed Congress, Wuhan, (TRCW'07), China, 108-110 pp.

37. Wang, X., G. Liu, Q. Yang, W. Hua, J. Liu and H. Wang. 2010. Genetic analysis on oil content in rapeseed (Brassica napus L.). Euphytica, 173: 17-24.

38. Zamani, M. and R. Choukan. 2005. Evaluation of combinig ability and genetic variance of maize line $\times$ tester crosses for determination of resistant sources to fusarium ear rot. Pajouhesh and Sazandegi, 66: 97-103 (In Persian). 


\title{
Estimation of Genetic Effects for Different Traits in Rapeseed (Brassica napus L.) using Line $\times$ Tester Crosses under Water-Stressed and Well-Watered Conditions
}

\author{
Mehdi Jamshidmoghadam ${ }^{1}$, Ezatoollah Farshadfar ${ }^{2}$ and Abduollah Najafi ${ }^{3}$ \\ 1 and 2- Ph.D. Student and Professor, Razi University \\ 3- Associated Professor, Razi University (Crossponding author: nadjaphy@ yahoo.com) \\ Recived: October 24, 2017 \\ Accepted: Septamber 30, 2018
}

\begin{abstract}
In order to estimate the effects of general (GCA) and specific (SCA) combining ability, heterosis and genetic control of different traits in rapeseed including yield and seed oil content, six lines of spring type and three testers Opera, Parade and Licord of winter type were crossed in 2014-2015. The 18 progenies and their parents were evaluated in a randomized complete block design at the Dryland Agricultural Research Institute (Sararood) under water-stressed (WS) and well-watered (WW) conditions in 2015-2016 cropping season.The results of line $\times$ tester analysis revealed that the GCA due to lines in all traits except for pod length in WW and the SCA due to line $\times$ tester in all of the traits except for seeds per pod and oil content in WS, and pod length in WW were significant for both environments. The genetic control of the traits was different in both conditions indicating that the gene effects were influenced by the environment for most studied traits. For seed yield, additive and non-additive effects were more important in WS and WW environments, respectively. Seed oil content had relatively low narrow sence heritability in both conditions. The most heterosis for seed yield was belonged to Comet $\times$ Opera $(168 \%$, WS $)$ and Amica $\times$ Licord $(171 \%, W W)$ hybrids The highest heterosis for seed oil content was observed in Shiralee $\times$ Opera hybrid in both environments $(12.6 \%$ in WS and 6.4\% in WW). The testers Opera and Licord were suitable to improve yield in WS and WW environments, respectively. The lines Comet and Dalgan had the highest GCA and the Dalgan $\times$ Licord hybrid had the highest SCA (Top cross) for seed yield in both conditions.
\end{abstract}

Keywords: Combining ability, Gene effect, Heritability, Heterosis 